\title{
Molecular Simulation Guided Constitutive Modeling on Finite Strain Viscoelasticity of Elastomers
}

\author{
Ying $\mathrm{Li}^{\mathrm{a}, \mathrm{d}, 1}$, Shan Tang ${ }^{\mathrm{b}, 1}$, Martin Kröger ${ }^{\mathrm{c}}$, Wing Kam Liu ${ }^{\mathrm{a}, *}$ \\ ${ }^{a}$ Department of Mechanical Engineering, Northwestern University, Evanston, IL 60208 \\ ${ }^{b}$ Department of Engineering Mechanics, Chongqing University, Shapingba, Chongqing, 400017 \\ ${ }^{c}$ Department of Materials, Polymer Physics, ETH Zurich, CH-8093 Zurich, Switzerland \\ ${ }^{d}$ Department of Mechanical Engineering \& Institute of Materials Science, University of Connecticut, Storrs, CT 06269, USA. Email: \\ yingli@engr.uconn.edu.
}

\begin{abstract}
Viscoelasticity characterizes the most important mechanical behavior of elastomers. Understanding the viscoelasticity, especially finite strain viscoelasticity, of elastomers is the key for continuation of their dedicated use in industrial applications. In this work, we present a mechanistic and physics-based constitutive model to describe and design the finite strain viscoelastic behavior of elastomers. Mathematically, the viscoelasticity of elastomers has been decomposed into hyperelastic and viscous parts, which are attributed to the nonlinear deformation of the cross-linked polymer network and the diffusion of free chains, respectively. The hyperelastic deformation of a cross-linked polymer network is governed by the cross-linking density, the molecular weight of the polymer strands between cross-linkages, and the amount of entanglements between different chains, which we observe through large scale molecular dynamics (MD) simulations. Moreover, a recently developed non-affine network model [Davidson and Goulbourne, 2013, J. Mech. Phys. Solids, 61, 1784] is confirmed in the current work to be able to capture these key physical mechanisms using MD simulation. The energy dissipation during a loading and unloading process of elastomers is governed by the diffusion of free chains, which can be understood through their reptation dynamics. The viscous stress can be formulated using the classical tube model [Doi and Edwards, 1986, The theory of polymer dynamics, Clarendon Press, Oxford]; however, it cannot be used to capture the energy dissipation during finite deformation. By considering the tube deformation during this process, as observed from the MD simulations, we propose a modified tube model to account for the finite deformation behavior of free chains. Combing the non-affine network model for hyperelasticity and modified tube model for viscosity, both understood by molecular simulations, we develop a mechanism-based constitutive model for finite strain viscoelasticity of elastomers. All the parameters in the proposed constitutive model have physical meanings, which are signatures of polymer chemistry, physics or dynamics. Therefore, parametric materials design concepts can be easily gleaned from the model, which is also demonstrated in this study. The finite strain viscoelasticity obtained from our simulations agrees qualitatively with experimental data on both un-vulcanized and vulcanized rubbers, which captures the effects of cross-linking density, the molecular weight of the polymer chain and the strain rate.
\end{abstract}

Keywords: Elastomer, Finite deformation, Viscoelasticity, Molecular dynamics, Constitutive modeling

\footnotetext{
${ }^{*}$ Corresponding author. E-mail: w-liu@ @orthwestern.edu (W.K.L.). Voice: +1-847-491-7094. Fax: +1-847-491-3915.

${ }^{1}$ These authors contributed equally to this work.
} 


\begin{tabular}{|c|c|}
\hline Nomenclature & $\begin{array}{l}\text { Definitions are given here in the following sequence: Roman alphabetical order followed } \\
\text { by Greek alphabetical order. Bold quantities denote vectors or tensors. }\end{array}$ \\
\hline$a_{\mathrm{pp} 0}, a_{\mathrm{pp}}$ & Tube diameter in reference/undeformed, current/deformed frame \\
\hline & Kuhn length of polymer chain \\
\hline B & Left Cauchy-Green tensor $\mathbf{F} \cdot \mathbf{F}^{T}$ \\
\hline$D_{c}$ & Diffusion coefficient of polymer chain \\
\hline $\mathscr{E}$ & Relaxation contribution to the kernel $\Upsilon$ \\
\hline G & Modulus entering $\sigma^{V}$ \\
\hline$G_{c}$ & Cross-linking modulus of polymer chains entering $\sigma^{E}$ \\
\hline$G_{e}$ & Plateau modulus induced by polymer chain entanglement entering $\sigma^{E}$ \\
\hline $\mathbf{F}$ & Deformation gradient tensor \\
\hline$F_{t}$ & Tensile force in primitive chain of tube model \\
\hline$I_{k}$ & $k$ th tensor invariant of $\mathbf{B}$ \\
\hline$k_{\mathrm{B}}$ & Boltzmann constant \\
\hline$L_{\mathrm{pp} 0}, L_{\mathrm{pp}}$ & Contour length of primitive path in reference/undeformed, current/deformed frame \\
\hline$N, N_{v}$ & Polymerization degrees of chains \\
\hline$N_{e}$ & Entanglement length, which is number of monomers between two entanglements \\
\hline$n_{v}$ & Number of polymer chains per unit volume \\
\hline $\mathbf{P}^{E}$ & Elastic part of first Piola-Kirchhoff stress tensor \\
\hline $\mathbf{P}^{V}$ & Viscous part of first Piola-Kirchhoff stress tensor \\
\hline$p$ & Mode number variable (odd integer) in tube model, $p=1,3, \ldots, \infty$ \\
\hline$R_{\mathrm{ee} 0}, R_{\mathrm{ee}}$ & End-to-end distance of polymer chain in reference/undeformed, current/deformed frame \\
\hline$s$ & Segment index/contour length variable along primitive chain \\
\hline$T$ & Temperature \\
\hline $\mathbf{v}_{k}$ & $k$ th eigenvector of $\mathbf{B}$ \\
\hline$W$ & strain energy density function $W=W(\mathbf{F})$ \\
\hline$\left\langle Z_{\text {kink }}\right\rangle,\left\langle Z_{\text {coil }}\right\rangle$ & Number of entanglements per chain given by kink assumption and random coil assumption \\
\hline & Argument in the generalized Mittag-Leffler function $E_{\alpha, 1}(x)$ \\
\hline $\boldsymbol{\sigma}^{E}$ & Elastic part of Cauchy stress tensor \\
\hline$\sigma^{V}$ & Viscous part of Cauchy stress tensor \\
\hline$\gamma$ & Shear strain \\
\hline$\lambda$ & Stretch ratio \\
\hline$\lambda_{\max }$ & Limiting network stretch \\
\hline$\lambda_{j}$ & Principle stretch, square root of $j$ th eigenvalue of $\mathbf{B}$ \\
\hline$\Lambda$ & Quantification of the rotation of the primitive chain contributing to viscous stress \\
\hline$\nu_{0}, \nu$ & Unit tangent vector of primitive chain in current/undeformed, reference/deformed frame \\
\hline$\Psi$ & Probability that chain segment $s$ remains in the tube of reptation at time $t$ \\
\hline$\phi$ & Crosslink functionality \\
\hline$\rho_{m}$ & Monomer mass density \\
\hline$\Upsilon$ & Integration kernel for the calculation of $\sigma^{V}$ \\
\hline$\tau_{d 0}, \tau_{d}$ & Disentanglement time of polymer chain in current/undeformed, reference/deformed frame \\
\hline$\xi$ & Friction coefficient of a polymer bead \\
\hline
\end{tabular}




\section{Introduction}

Elastomers consist of long polymer chains joined together by chemical bonds through cross-linkers. They are usually capable of recovering their original shapes after finite deformation due to covalent crosslinkages. Under equilibrium conditions, long polymer chains making up an elastomer are irregularly coiled together. However, when the elastomer is under tension, polymer chains tend to stretch out and straighten along the pulling direction. Upon unloading, the chains return to their original compact and random arrangement. During such a loading-unloading process, energy dissipates due to friction between polymer chains and the elastomer exhibits viscoelastic behavior, as illustrated in Fig. 1. Examples of elastomers include polyisoprene (natural rubber), polybutadiene (butadiene rubber), polychloroprene (chloropene rubber) and the copolymer comprised of butadiene and acrylonitrile (nitrile rubber). These materials have a broad range of applications in engineering and industry.
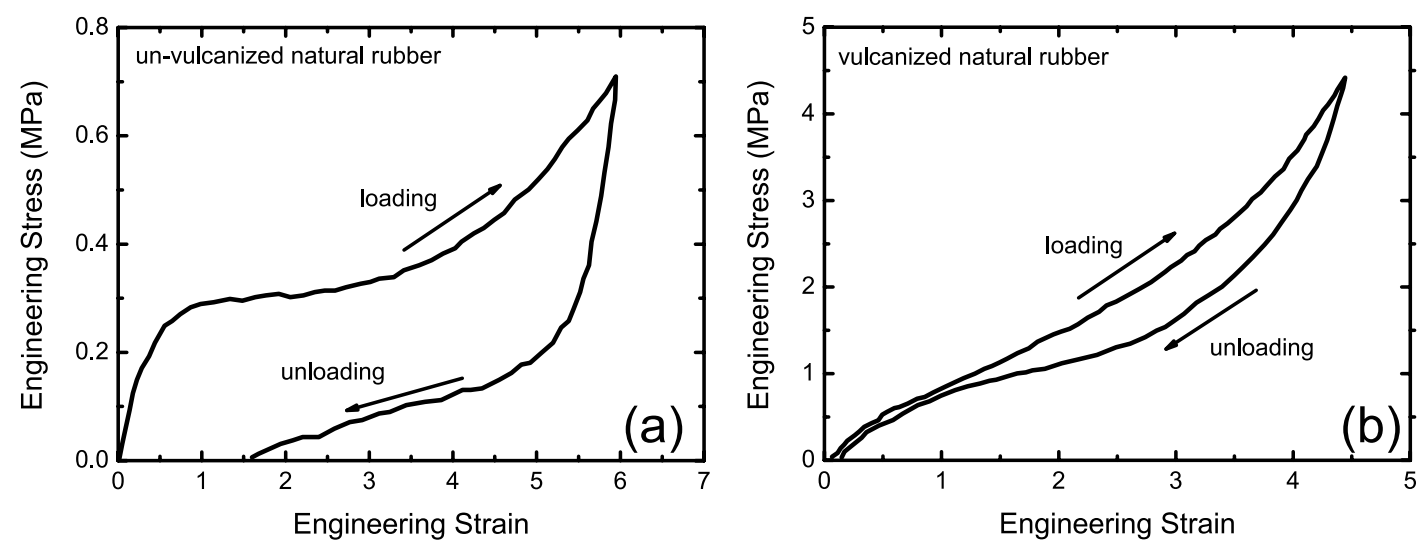

Figure 1: Experimental results on uniaxial stress-strain curves during loading and subsequent un-loading of (a) un-vulcanized and (b) vulcanized natural rubber (NR) with strain rate $0.003 s^{-1}$ at room temperature. Results taken from Amnuaypornsri et al. (2012).

Due to differences in the degree of cross-linking, the viscoelastic behavior of un-vulcanized natural rubber $(\mathrm{NR})^{1}$ can be very distinct from that of vulcanized NR (Fig. 1). For un-vulcanized NR under uniaxial tension (Fig. 1a), the stress initially increases linearly with strain during the elastic deformation stage, followed by a strain-softening stage with a stress plateau. Upon increasing the strain further, the stress starts to increase rapidly, reminiscent of strain-hardening behavior. When the applied tension is released, the stress drops rather quickly and the polymer chains tend to return to a coiled equilibrium state that might be very different from the initial state. Therefore, the stress-strain curve of un-vulcanized NR exhibits pronounced hysteretic behavior, representing large energy dissipation. Contrary to the behavior of un-vulcanized NR, the vulcanized NR exhibits only the strain-hardening behavior without showing strain-softening (cf. Fig. 1b). During the unloading stage, the stress slowly reduces with decreasing strain, signaling a much smaller hysteresis with less energy dissipation, because of the constraints applied through the cross-linkages. Moreover, the elastic modulus of vulcanized NR is about 20-30 times larger than that of un-vulcanized NR due to large differences in cross-linking densities. The above stress-strain curves for un-vulcanized and vulcanized NRs characterize the most important mechanical behavior of elastomers, which can greatly affect their

\footnotetext{
${ }^{1}$ Although the NRs are un-vulcanized, it is experimentally found that they have naturally occurred (pseudo) endlinkings (Amnuaypornsri et al., 2012). Therefore, these un-vulcanized NRs exhibit a low degree of cross-linking.
} 
performance in applications. However, a reliable constitutive model that is able to capture these qualitative differences in mechanical behavior for un-vulcanized and vulcanized NRs simultaneously does not presently exist.

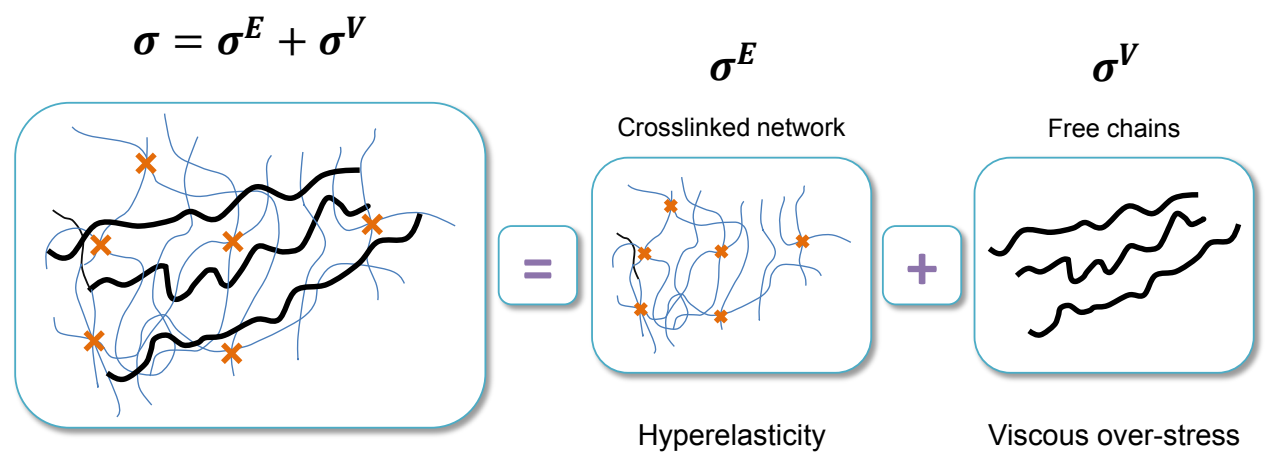

Figure 2: Decomposition of an elastomer into a cross-linked polymer network with free chains. The hyperelastic stress $\boldsymbol{\sigma}^{E}$ and viscous stress $\sigma^{V}$ are contributed by the nonlinear deformation of the cross-linked network and diffusion of free chains, respectively. Therefore, the mechanical response of the elastomer can be formulated as $\boldsymbol{\sigma}=\boldsymbol{\sigma}^{E}+\boldsymbol{\sigma}^{V}$.

To rationalize the viscoelastic behavior of elastomers, their stress response is usually decomposed into two parts: (1) a hyperelastic part and (2) a viscous part (schematically depicted in Fig. 2). The elastic part is assumed to originate from the connectivity and stretching of the polymer chain's backbone, while the viscous part is assumed to originate from polymer diffusion. If the polymer chains are short or "oligomeric", their dynamics are governed by a friction coefficient. According to the Rouse theory (Rouse Jr, 1953), which was originally developed for unentangled polymer solutions, the polymer's zero-rate viscosity $\eta_{0}$ is linearly proportional to the polymerization degree of the chain $N$ as $\eta_{0} \sim N$, which has been widely confirmed by experiments and molecular simulations for both solutions and melts (Kröger et al., 1993; Li et al., 2013). However, if $N$ exceeds a system-specific characteristic value, $N_{e}$, the polymer chains are highly entangled. Due to the connectivity and uncrossability of polymer chains, the dynamics of highly entangled polymer chains are hindered by their entanglements ("topological constraints"). In the early theoretical study by Doi and Edwards (1986), entanglements were assumed to effectively restrict the lateral motion of individual chains into a tube-like region. Within this picture, a chain can only move back and forth, or reptate, within its tube, instead of moving randomly in space. It can leave the tube solely through its two opposing ends. After some sufficiently long period of time, which is denoted as disentanglement time $\tau_{d}$, the chain will completely escape the original tube and form a new tube with other chains from its neighborhood; at this time the relaxation of a polymer chain has been accomplished. The above picture for entangled polymer chain dynamics has been coined the tube model, which is considered to be the most successful theory in polymer physics from the past thirty years.

A huge number of modified reptation models have been proposed to quantitatively capture experimental findings. According to the tube theory, the complex three dimensional behavior of the polymer chains can be simplified into a one-dimensional problem as the chain moves inside the tube in a one-dimensional diffusive manner. Pioneered by De Gennes (1979); Doi and Edwards (1986), the relaxation and viscosity of entangled polymers can be theoretically studied through the tube model and their viscosity $\eta_{0}$ scales with $N$ as $\eta_{0} \sim N^{3}$, which agrees reasonably well with experimental observations. In later work, the contour length fluctuation (CLF) and constrained release (CR) effects, which have been observed from the real polymer systems, were incorporated into the original tube model and lead to $\eta_{0} \sim N^{3.4}$ (McLeish, 2002). 
In the original tube theory, the contour length of the primitive path was assumed to be fixed at its mean value $L_{\mathrm{pp}}$. In reality, however, the path length fluctuates around this mean value with root-mean-squared fluctuation on the order of $\delta L_{\mathrm{pp}} \sim L_{\mathrm{pp}}\left(N_{e} / N\right)^{2}$ (McLeish, 2002), where $N_{e}$ is the number of monomers between two adjacent entanglements. Therefore, the CLF mechanism manifests itself in the dynamics of moderately long chains and becomes negligible for extremely long chains. In additional to the CLF effect, the movement of neighboring chains can release the lateral constraints to the motion of the tracer chain, leading to the dilation or reorganization of its surrounding tubes, which is the so-called CR mechanism (McLeish, 2002). In contrast to the CLF, which is a single-chain phenomenon, the CR is a cooperative phenomenon between different chains, but both of them hasten the relaxation process of chains. Taking these important physical mechanisms into account, various theoretical models have been developed for the linear viscoelastic properties of polymers (McLeish, 2002; Watanabe, 1999; Schweizer and Curro, 1997; Likhtman and McLeish, 2002), which has resulted in significant improvement when compared with experiments. The related theoretical studies have been reviewed in the literature on the subject (McLeish, 2002; Watanabe, 1999; Schweizer and Curro, 1997; Li et al., 2013). Most of the above mentioned studies are applicable for the linear viscoelastic behaviors of polymers without considering the finite strain viscoelasticity.

For the majority of model systems in rubber science, more than $90 \%$ of the free energy has an entropic origin (Edwards and Vilgis, 1988). Its elasticity thus mainly depends on the entropic force with its strength proportional to $k_{\mathrm{B}} T$, where $k_{\mathrm{B}}$ and $T$ are the Boltzmann constant and temperature, respectively. The tube concept has been used to explain why the elastic modulus of rubber is independent of the strand length in the polymer network (Edwards (1967)). Subsequent work on the contributions of entanglements and cross-links on the elastic response of a cross-linked polymer network have been derived through statistical mechanics formulations (Edwards and Vilgis, 1988), which were based on the affine deformation assumption. Following a similar idea, Rubinstein and Panyukov (1997) have established an affine length scale $R_{\text {aff }}$, separating the deformation of the elastomer into two regimes: one is the solid, elastic and affine deformation on large scales, and the other is the liquid-like nonaffine deformation, which is on smaller scales. Within this model, the nonlinear elasticity of elastomers is induced by nonaffine deformation. The same authors combined and generalized several successful ideas into a new molecular mechanism-based nonlinear elastic model for elastomers (Rubinstein and Panyukov, 2002) that incorporates the concepts of the tube model as well as CLF and CR effects. The predicted results from this model agree well with both experimental and computer-simulation findings (Rubinstein and Panyukov, 2002).

It is important to emphasize that the Rubinstein-Panyukov model for elastomers works well only for the case of uniaxial tension deformation and that it does not provide a unified strain energy density function to describe the different deformation states. Moreover, the extension limitation of the polymer chain has been ignored. To overcome these issues, Arruda and Boyce (1993) has simplified the underlying microstructure of the elastomer into an eight-chain cross-linked cube and derived a unified strain energy density function for different deformation states, with each chain characterized by Langevin (non-Gaussian) behavior. The proposed model simultaneously works well for uniaxial tension, equibiaxial extension, uniaxial compression and pure shear. Nevertheless, the Arruda-Boyce model does not consider the entanglement effect and the real molecular structure of the elastomer has been oversimplified. Recently, Davidson and Goulbourne (2013) have combined the concepts of the Rubinstein-Panyukov and Arruda- Boyce models and developed a new physics-based model for the nonlinear elasticity of elastomers, the so-called non-affine network model. The non-affine network model potentially captures the cross-linking density effect, entanglement effect and deformation-state-dependent response of an elastomer under finite deformation.

To tackle the finite strain viscoelastic properties of unfilled or filled elastomers, phenomenological constitutive models have been developed by Simo (1987); Govindjee and Simo (1992); Lion (1996) based on internal stress-like variables; the resulting constitutive models have been used to fit experimental results. 
Lubliner (1985); Lion (1997); Keck and Miehe (1997) have developed phenomenological models based on tensorial strain variables. Sidoroff (1974); Le Tallec et al. (1993); Govindjee and Reese (1997); Reese and Govindjee (1998) have introduced and developed a form of finite viscoelasticity based on a multiplicative decomposition of the deformation gradient. The total deformation gradient has been decomposed into two parts: elastic and inelastic (or viscous). However, the main use of these phenomenological constitutive models is to fit existing experiment data since microscopic details about the underlying physical mechanisms do not enter their parameters. In an attempt to overcome this issue, Bergström and Boyce (1998); Miehe and Göktepe (2005) have borrowed the concepts of the tube model (De Gennes, 1979; Doi and Edwards, 1986) to develop their constitutive models for finite strain viscoelasticity, based on the multiplicative decomposition of the deformation gradient. Still, these models exhibit several internal variables without any obvious physical meaning, and can thus also be considered to fall into the class of phenomenological models with a micromechanics concept. More recently, Tang et al. (2012); Li et al. (2012b) have developed a mechanisticbased constitutive model for viscoelastic properties of elastomers by decomposing the stress response into an elastic and viscous part. The elastic and viscous responses are captured by the Arruda-Boyce (eightchain) (Arruda and Boyce, 1993) and tube (Doi and Edwards, 1986) models, respectively. For this model, all the related parameters entering the constitutive model have a physical foundation and can be predicted or informed by either molecular simulations or experiments (Li et al., 2012b, 2013). Such a model, in which the material law parameters represent signatures from polymer chemistry, physics and dynamics, should have advantages over the existing phenomenological models as it provides parametric materials design principles for elastomers. Here we should emphasize that the ideal elastomer is assumed to be isotropic and homogeneous, although the un-vulcanized NR can demonstrate inhomogeneity (Toki et al., 2008).

While this mechanistic-based model has already been used to capture the uniaxial loading and unloading behaviors for vulcanized NR (Tang et al., 2012), it fails to capture the strain-softening behaviors of un-vulcanized NR during the same process (cf. Fig. S2b in ESI) (Li et al., 2012b). It could therefore not be used to design un-vulcanized and vulcanized rubbers simultaneously. To improve on this, molecular simulations can play an important role by verifying new and existing theoretical models, but also in guiding the development of new theories and exploring unexpected physical phenomena. As we'll show below, by rigorously validating and verifying the underlying assumptions made in the constitutive models through molecular simulations, the mechanistic-based constitutive models are considerably improved upon by: (i) adding new physical mechanisms revealed from our MD simulations and (ii) removing unnecessary assumptions. Eventually, the proposed constitutive model can be further applied to design the finite strain viscoelastic behaviors of elastomers with different cross-linking densities, entanglement densities, and polymerization degrees, to name a few.

Comparing with the previous studies on the finite strain viscoelasticity of elastomers, the major contributions of this work are:

- A new constitutive model for the finite strain viscoelastic behavior of elastomers has been created by decomposing the elastomer into a cross-linked network with superimposed free chains, denoting the hyperelastic and viscous responses, respectively. The state-of-the-art continuum models for hyperelastic and viscous behaviors of polymer chains have been adopted and developed further.

- All the important assumptions in the continuum model have been tested through large scale molecular dynamics (MD) simulations. Existing theoretical models have been tested and verified by the MD simulations. New physical mechanisms have been added into the constitutive model through the observations from MD simulations.

- All the material law parameters entering the constitutive model have actual physical meaning. Each parameter can be directly obtained through MD simulations or measured by experiments. 
- Finite strain viscoelasticity of un-vulcanized and vulcanized rubbers is simultaneously captured by the constitutive model, which has been further verified by comparing with existing experimental results.

The manuscript is organized as follows. The proposed constitutive model for elastomers has been summarized in Section 2; the key material law parameters are also highlighted and explained in detail. Section 3 describes the theoretical background for Arruda-Boyce and non-affine network models and these hyperelastic models are tested by comparing with stress-strain curves obtained from MD simulations of cross-linked polymer networks. The MD simulation method is described in detail in the Electronic Supplementary Information (ESI). The underlying assumptions made in these hyperelastic models are verified directly by inspecting the MD simulation results. The theoretical formulation for the viscous behavior of free polymer chains, based on the tube theory, are introduced in Section 4; the theory is further extended to the finite deformation range based on observations from MD simulations. In Section 5, the constitutive model is built by combining the non-affine network model with the refined tube model. Experimental results on the stressstrain curves of un-vulcanized and vulcanized rubbers are used to verify the proposed constitutive model. Parametric material design concepts based on the proposed constitutive model for finite strain viscoelasticity of elastomers will also be exemplified. Section 6 concludes the present study.

\section{Summary of Constitutive Model}

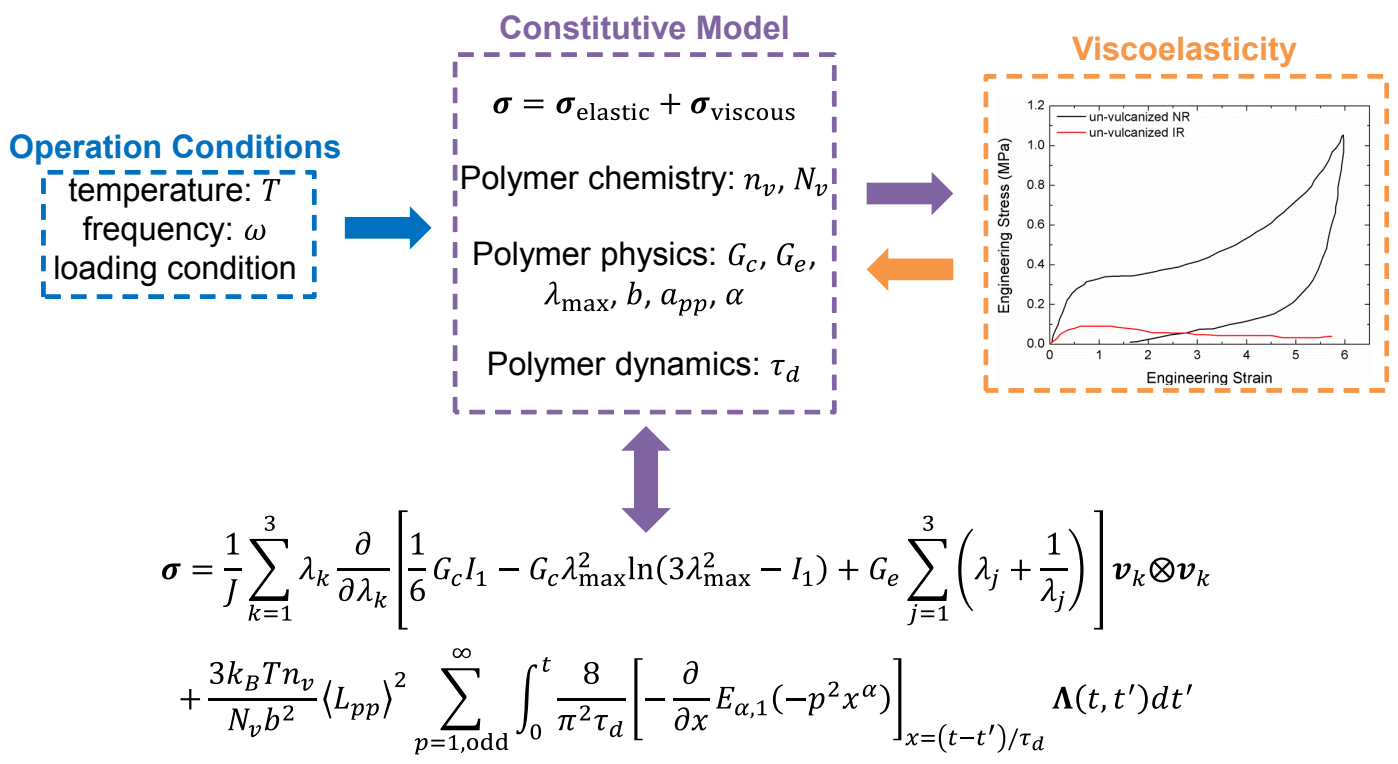

Figure 3: Summary of the proposed constitutive model for viscoelasticity of elastomers. Elastic and viscous contributions of the stress tensor are denoted by $\boldsymbol{\sigma}^{E}$ and $\boldsymbol{\sigma}^{V}$ in the text part. In this schematic, tube diameter $a_{\mathrm{pp}}$ and disentanglement time $\tau_{d}$ stand for their values in the undeformed state, both are predicted to depend on the deformation state. $\mathbf{v}_{k}$ (all of length unity) are eigenvectors corresponding to their eigenvalues $\lambda_{k}^{2}$ of the Cauchy-Green tensor, with subscript $k=1,2$ and 3 denoting the $x, y$ and $z$ directions, respectively. $J$ is the determinate of the deformation gradient $\boldsymbol{F} . I_{1}$ is the first invariant of the Cauchy-Green tensor. $k_{\mathrm{B}}$ and $T$ are the Boltzmann constant and temperature, respectively. See the main text for more details about the material law parameters in the proposed constitutive model. 
In Fig. 3, we summarize the proposed constitutive model for finite strain viscoelasticity of elastomers and highlight the key material law parameters. Our constitutive model includes nine parameters in total. According to their physical meanings, they are classified into three different categories: polymer chemistry, physics and dynamics. These material parameters are discussed in detail in the following list and are summarized in Tab. 1:

- $n_{v}$ is the number of free chains per unit volume, which belongs to the polymer chemistry. It is determined during the material synthesis.

- $N_{v}$ is the polymerization degree of free chains, which belongs to the polymer chemistry. It is also determined during the material synthesis.

- $G_{c}$ is the cross-linking modulus from hyperelastic part, and is a signature of polymer physics. According to the work by (Davidson and Goulbourne, 2013), $G_{c}=\rho_{m} k_{\mathrm{B}} T g^{2}(1-2 / \phi) / N$, where $\rho_{m}$ is the monomer mass density. $\phi$ and $N$ are the functionality of cross-linkers and polymerization degree of the cross-linked chains. $g$ denotes the affinity of the polymer chain length during deformation and is considered to be a constant, as discussed in ESI Section 1.

- $G_{e}$ is the entanglement modulus from hyperelastic part, and is a signature of polymer physics. According to the work by Doi and Edwards (1986), $G_{e}=\frac{4}{5} \rho_{m} k_{\mathrm{B}} T / N_{e}=\frac{4}{5} G_{c} N / N_{e}$, where $N_{e}$ is the entanglement length, representing the number of monomers between two adjacent entanglements.

- $\lambda_{\max }$ characterizes the limited extensibility of chains for the hyperelastic part, and is a signature of polymer physics. According to the work by Davidson and Goulbourne (2013), $\lambda_{\max }=\sqrt{N / g^{2}(1-2 / \phi)}$.

- $b$ is the Kuhn length for the free chains of the viscous part, and is a signature of polymer physics. It is related to the chain's end-to-end distance, $R_{\mathrm{ee}}$ as $R_{\mathrm{ee}}^{2}=N_{v} b^{2}$ (Doi and Edwards, 1986), when the chain follows the Gaussian statistics (coiled configuration). It should be noted that $N_{v}$ in this expression for $R_{\mathrm{ee}}$ is the number of Kuhn steps, constructed so that $N_{v} b$ is the maximum extension of the chain and $R_{\mathrm{ee}}^{2}$ the mean squared end-to-end distance of the free chain, that is available from experiments.

- $a_{\mathrm{pp}}$ is the tube diameter of free chains for the viscous part, and is a signature of polymer physics. It can be estimated via near-field microscopic measurements or dynamic scattering techniques by comparing with theoretical predictions (Fetters et al., 1994; Heinrich et al., 2004; Keshavarz et al., 2015). With the help of primitive path analysis (Kröger, 2005), $a_{\mathrm{pp}}$ can be efficiently calculated based on the atomistic configurations of polymer chains as $a_{\mathrm{pp}}=R_{\mathrm{ee}}^{2} / L_{\mathrm{pp}}$ (Doi and Edwards, 1986), which has been found to yield excellent agreement with the experimentally derived value ( $\mathrm{Li}$ et al., 2012b). Here $L_{\mathrm{pp}}$ is the primitive chain length, directly measurable during the primitive path analysis. More details are given in ESI Section 4.

- $\alpha$ is the fractional order parameter for the relaxation of free chains, and is a signature of polymer physics. The fractional order parameter $\alpha$ can be directly measured by the chain's survival probability function $\Psi(s, t)$ (cf. Eq. (23)) computed from molecular simulations (Stephanou et al., 2010) or calibrated through relaxation spectrum of polymers (Li et al., 2012b).

- $\tau_{d}$ is the disentanglement time for the free chains, which is the polymer dynamics parameter. According to the tube theory (Doi and Edwards, 1986), we have $\tau_{d}=L_{\mathrm{pp}}^{2} / \pi^{2} D_{c}$, where $D_{c}$ is the diffusion coefficient of free chains. In our previous study (Li et al., 2012b), we have demonstrated that $L_{\mathrm{pp}}$ and $D_{c}$ can be directly obtained through our large scale coarse-grained molecular simulations. 
Table 1: List of material parameters in proposed constitutive model for finite viscoelasticity of elastomers. Details about these parameters are referred to the main text and ESI Section 1.

\begin{tabular}{l|l|l|l}
\hline \hline \multicolumn{3}{c}{ Summary of Material Parameters } \\
\hline Category & Parameter & Definition & Measurement \\
\hline \multirow{2}{*}{ Polymer Chemistry } & $n_{v}$ & number of chains per unit volume & determined during synthesis \\
& $N_{v}$ & polymerization degree & determined during synthesis \\
\hline & $G_{c}$ & cross-linking modulus & $G_{c}=\rho_{m} k_{\mathrm{B}} T g^{2}(1-2 / \phi) / N$ \\
& $G_{e}$ & entanglement modules & $G_{e}=\frac{4}{5} \rho_{m} k_{\mathrm{B}} T / N_{e}=\frac{4}{5} G_{c} N / N_{e}$ \\
\multirow{3}{*}{ Polymer Physics } & $\lambda_{\max }$ & chain extensibility & $\lambda_{\max }=\sqrt{N / g^{2}(1-2 / \phi)}$ \\
& $b$ & Kuhn length & $b^{2}=R_{\mathrm{ee}}^{2} / N_{v}$ \\
& $a_{\mathrm{pp}}$ & tube diameter & $a_{\mathrm{pp}}=R_{\mathrm{ee}}^{2} / L_{\mathrm{pp}}$ \\
& $\alpha$ & fractional order parameter & measured by $\Psi(s, t)$ \\
\hline Polymer Dynamics & $\tau_{d}$ & disentanglement time & $\tau_{d}=L_{\mathrm{pp}}^{2} / \pi^{2} D_{c}$ \\
\hline \hline
\end{tabular}

From the above list, it can be observed that the majority of the parameters in the proposed constitutive model are related to the polymer physics. For the hyperelastic behaviors of elastomers, these three parameters, cross-linking modulus $G_{c}$, entanglement modulus $G_{e}$ and chain extensibility $\lambda_{\max }$ are determined by the microstructure of elastomers, such as cross-linking density, entanglement density, and molecular weight of the polymer strand between cross-linkages. However, due to the lack of exact information about the cross-linked network in elastomers, these parameters are usually not easily directly determined through their microstructures. As demonstrated in the present study (Section 3.2), these polymer physics parameters can be easily calibrated through the uniaxial tension stress-strain curve given by molecular simulations or experiments (Davidson and Goulbourne, 2013), which is the method that we will use when comparing the experimental results on stress-strain curves of un-vulcanized and vulcanized NRs with the proposed constitutive model. Furthermore, we have discussed underlying microscopic expressions for quantities like $G_{c}$, $G_{e}$, or $\lambda_{\max }$. A larger amount of experimental data is needed to test these microscopic expressions, but underlying microscopic expressions are certainly helpful in the design process.

The rest of the polymer physics parameters are related to the tube behavior of free chains. In our large scale coarse-grained molecular simulations (Li et al., 2012b), we have demonstrated that these parameters can be determined by these simulations. The values of $b, a_{\mathrm{pp}}$ and $\tau_{d}$ for polyisoprene (major component of NR) are found to be in good agreement with experimental measurements. Thus, these reported values will be used in our constitutive model for predicting the viscous energy dissipation of un-vulcanized and vulcanized NRs during the uniaxial loading-unloading process (cf. Section 5.2).

According to above discussions, we can see that all the material law parameters in our proposed constitutive model have clear physical meaning. Therefore, under the given operation conditions, for example, temperature $T$ or frequency $\omega$ and loading condition, the constitutive model can be used to predict mechanical behaviors of elastomers, as these parameters can be directly obtained through molecular simulations or measured by experiments. On the other hand, these parameters are also materials design parameters, as they are signatures of polymer chemistry, physics and dynamics. Thus, the present constitutive model can be further applied for designing materials with desired viscoelastic properties. In the following sections, we will demonstrate how the constitutive model is developed and guided by molecular simulations.

Finally, we should emphasize that the entanglement modules $G_{e}$ should be attributed to the entanglement 
effect in the cross-linked polymer network, and classified into the hyperelastic part. Viscoelasticity is the property of materials that behave both elastic and viscous when undergoing deformation. The elasticity does not depend on time/frequency while the viscosity does. According to this definition, we separate the viscoelasticity of elastomers into two parts: one is the hyperelastic part, attributed to the deformation of the cross-linked network, and the other one is the viscous part, attributed to the diffusion of free chains. For the cross-linked network, both cross-links and entanglements should play important roles, as they can impose the constraints on polymer chains when the chains are deformed. Also, from experimental results on VHB 4905 rubber (Fig. S2 in the ESI), the entanglement contribution $\left(G_{e}\right)$ should be considered. Otherwise the strain-softening behavior cannot be captured in the hyperelastic deformation. On the other hand, both the storage modulus $G^{\prime}$ and loss modulus $G^{\prime \prime}$ of the viscous part (free chains) exhibit time/frequency dependent behaviors. For instance, $G^{\prime} \sim \omega^{2}$ and $G^{\prime \prime} \sim \omega$ in the low frequency regime $\left(\omega<\tau_{d}^{-1}\right)$ (Li et al., 2012b). Since $G_{e}$ does not depend on the imposed frequency, it is different from the storage modulus $G^{\prime}$ or loss modulus $G^{\prime \prime}$ of the free chains. In this way, we believe that $G_{e}$ should not be attributed to the viscous part.

\section{Hyperelastic constitutive model for cross-linked polymer network}

\subsection{Theoretical background}

The nonlinear elasticity of a cross-linked polymer network is typically characterized by a hyperelastic constitutive model, which derives from a strain energy density function $W$. According to the definition of $W$, the material models can be classified as phenomenological or mechanistic models. The phenomenological models can be used to describe and fit experimentally observed behaviors, while mechanistic models are derived from the arguments about the underlying physics of the materials during the deformation. Along this line, Mooney-Rivlin (Mooney, 1940; Rivlin, 1948), Ogden (1972) and Yeoh (1993) are the most widely used phenomenological modes for nonlinear elasticity. For the mechanistic models, there are the ArrudaBoyce model (Arruda and Boyce, 1993), slip-link model (Edwards and Vilgis, 1986), extended tube model (Kaliske and Heinrich, 1999), ABGI model (Meissner and Matějka, 2003), micro-sphere model (Miehe et al., 2004), and the non-affine network model (Davidson and Goulbourne, 2013). These different mechanistic models have been briefly compared by Davidson and Goulbourne (2013) and were found to be capable of capturing the same stress-strain behavior of VHB 4905 elastomers under uniaxial tension, including both strain softening and hardening; an exception is the Arruda-Boyce model (cf. Fig. S2a in ESI). We choose the Arruda-Boyce and non-affine network models for nonlinear elasticity of cross-linked networks as they have the least material parameters to be determined. There are only two and three material parameters needing determination for the Arruda-Boyce and non-affine network models, respectively. The other mechanistic models require four or more material parameters and some of them are uneasy to be calibrated through simulations or experiments.

According to the Arruda-Boyce model (Arruda and Boyce, 1993), revisited in ESI Section 1 and expressed by using the first five terms of the inverse Langevin function, the elastic energy density is given by

$$
W^{\mathrm{AB}}(\mathbf{F})=G_{c} \sum_{i=1}^{5} \alpha_{i} \beta^{i-1}\left(I_{1}^{i}-3^{i}\right),
$$

where $\beta=1 / \lambda_{\max }^{2}$, and $\alpha_{1}=1 / 2, \alpha_{2}=1 / 20, \alpha_{3}=11 / 1050, \alpha_{4}=19 / 7000, \alpha_{5}=519 / 673750$. $\mathbf{F}$ is the deformation gradient and $I_{1}$ is the first invariant of the left Cauchy-Green deformation tensor. $G_{c}=\rho_{m} k_{\mathrm{B}} T / N$ represents the cross-link modulus, $\rho_{m}$ and $N$ representing the monomer mass density and polymerization degree of the strand between cross-linkages, respectively. $\lambda_{\max }=\sqrt{N}$, defined as the 
chain's final length divided by its initial length, characterizes the finite-extensibility of the chain (ESI Section 1). Since $W^{\mathrm{AB}}$ depends on $I_{1}$ alone, Eq. (1) yields the Cauchy stress

$$
\boldsymbol{\sigma}^{E}=p_{\text {hydro }} \mathbf{1}+2 \frac{\partial W^{\mathrm{AB}}}{\partial I_{1}} \mathbf{B}=p_{\text {hydro }} \mathbf{1}+2 G_{c}\left(\sum_{i=1}^{5} i \alpha_{i} \beta^{i-1} I_{1}^{i-1}\right) \mathbf{B}
$$

where $p_{\text {hydro }}$ and $\mathbf{1}$ are the hydrostatic pressure and identity tensor, respectively. To simplify the expression, we assume that the 1, 2 and 3 directions are aligned with the principal-1, -2 and -3 directions in the following formulation. When the elastomer is under uniaxial tension with stretch ratio $\lambda$, we will have $\lambda_{1}=\lambda$, and $\lambda_{2}=\lambda_{3}=1 / \sqrt{\lambda}$ due to the incompressibility and isotropy of the material, and $B_{11}-B_{22}=\lambda_{1}^{2}-\lambda_{2}^{2}$. Then, the corresponding mechanical response following from Eq. (1) and Eq. (2) can be formulated as

$$
\sigma_{11}^{E}-\sigma_{22}^{E}=2 G_{c}\left(\lambda^{2}-\frac{1}{\lambda}\right)\left[\sum_{i=1}^{5} i \alpha_{i} \beta^{i-1}\left(\lambda^{2}+\frac{2}{\lambda}\right)^{i-1}\right] .
$$

because $I_{1}=\lambda^{2}+2 / \lambda$ in that case. Considering an equibiaxial tension with $\lambda_{1}=\lambda_{2}=\lambda$ and $\lambda_{3}=1 / \lambda^{2}$, the stress response is given by

$$
\sigma_{11}^{E}-\sigma_{33}^{E}=2 G_{c}\left(\lambda^{2}-\frac{1}{\lambda^{4}}\right)\left[\sum_{i=1}^{5} i \alpha_{i} \beta^{i-1}\left(2 \lambda^{2}+\frac{1}{\lambda^{4}}\right)^{i-1}\right] .
$$

For a simple shear deformation, the deformation gradient and left Cauchy-Green tensors are

$$
\mathbf{F}=\left[\begin{array}{lll}
1 & \gamma & 0 \\
0 & 1 & 0 \\
0 & 0 & 1
\end{array}\right], \quad \mathbf{B}=\left[\begin{array}{ccc}
1+\gamma^{2} & \gamma & 0 \\
\gamma & 1 & 0 \\
0 & 0 & 1
\end{array}\right]
$$

where $\gamma$ is the engineering shear strain and $I_{1}=3+\gamma^{2}$. According to Eq. (2), the Cauchy stress tensor becomes

$$
\boldsymbol{\sigma}^{E}=p_{\text {hydro }} \mathbf{1}+2 G_{c}\left[\sum_{i=1}^{5} i \alpha_{i} \beta^{i-1}\left(3+\gamma^{2}\right)^{i-1}\right] \mathbf{B},
$$

Note that in the Arruda-Boyce model, the entanglements between different polymer chains are ignored. Thus, the Arruda-Boyce model cannot capture the entanglement effect. Simple rational approximates to the inverse Langevin function and corresponding variants of the elastic energy density in Eq. (1) that are more suitable for strong flows and deformations had been recently derived in Kröger (2015).

To include the entanglement effect during the nonlinear deformation process, Davidson and Goulbourne (2013) have combined the Arruda and Boyce (1993) and Rubinstein and Panyukov (2002) models to develop a non-affine network model. As mentioned in the introduction, the Arruda-Boyce model captures the deformation-state-dependent response with the extension limitation of polymer chains, and the RubinsteinPanyukov model considers both the non-affine deformation and entanglement effects. Therefore, the nonaffine network model (Davidson and Goulbourne, 2013) can capture the cross-linking density effect, entanglement effect, and the limited extension of polymer chains during finite deformation. Its strain energy density is formulated based on the principal stretches $\lambda_{j}$ (see more details in the ESI Section 1)

$$
W^{\text {nonaffine }}=\frac{1}{6} G_{c} I_{1}-G_{c} \lambda_{\max }^{2} \ln \left(3 \lambda_{\max }^{2}-I_{1}\right)+G_{e} \sum_{j=1}^{3}\left(\lambda_{j}+\frac{1}{\lambda_{j}}\right),
$$


Table 2: Simulated cross-linked polymer networks. $N$ is the polymerization degree of the chain between two adjacent cross-linkers; $n_{\mathrm{ch}}$ and $n_{\mathrm{ck}}$ denote the number of polymer chains and cross-linkers, respectively, $L$ is the simulation box side length, and $\langle\phi\rangle$ is the cross-linking functionality of the cross-linker. $\left\langle R_{\mathrm{ee}}^{2}\right\rangle^{1 / 2}$ is the measured mean squared end-to-end distance of cross-linked chains. $L_{\mathrm{pp} 0}$ and $a_{\mathrm{pp} 0}$ are the mean contour length of the primitive chain and tube diameter, respectively. $\left\langle Z_{\mathrm{kink}}\right\rangle$ and $\left\langle Z_{\text {coil }}\right\rangle$ characterize the number of entanglements per chain defined by the kink and coil assumptions, respectively. Details about the primitive path analysis are given in the ESI Section 4 to calculate $L_{\mathrm{pp} 0}, a_{\mathrm{pp} 0},\left\langle Z_{\mathrm{kink}}\right\rangle$ and $\left\langle Z_{\text {coil }}\right\rangle$.

\begin{tabular}{rrrrcccccc}
\hline \multicolumn{1}{c}{$N$} & \multicolumn{1}{c}{$n_{\text {ch }}$} & \multicolumn{1}{c}{$n_{\text {ck }}$} & \multicolumn{1}{c}{$L$} & $\langle\phi\rangle$ & $\left\langle R_{\text {ee }}^{2}\right\rangle^{1 / 2}$ & $L_{\mathrm{pp} 0}$ & $a_{\mathrm{pp} 0}$ & $\left\langle Z_{\text {kink }}\right\rangle$ & $\left\langle Z_{\text {coil }}\right\rangle$ \\
\hline 20 & 5000 & 2500 & 48.546 & 3.97 & 5.574 & 5.917 & 5.612 & 0.163 & 0.134 \\
40 & 2500 & 1250 & 48.396 & 3.97 & 8.193 & 9.456 & 7.565 & 0.653 & 0.341 \\
100 & 1000 & 500 & 48.310 & 3.97 & 13.047 & 18.638 & 9.621 & 2.104 & 1.051 \\
200 & 500 & 250 & 48.290 & 3.91 & 17.870 & 32.925 & 10.070 & 4.180 & 2.407 \\
500 & 200 & 100 & 48.248 & 3.89 & 28.992 & 70.759 & 12.145 & 9.420 & 4.967 \\
1000 & 100 & 50 & 48.266 & 3.74 & 39.249 & 127.557 & 12.214 & 17.160 & 9.572 \\
\hline \hline
\end{tabular}

where $G_{c}$ and $G_{e}$ denote the moduli contributed by the cross-linking and entanglement of polymer chains, respectively. The detailed definitions of $G_{c}, G_{e}$ and $\lambda_{\max }$ are given in ESI Section 1.

According to the strain energy density function for the non-affine network model given by Eq. (7), the Cauchy stress is

$$
\sigma_{11}^{E}-\sigma_{22}^{E}=\frac{1}{3} G_{c}\left(\lambda^{2}-\frac{1}{\lambda}\right)\left(\frac{\lambda^{2}+\frac{2}{\lambda}-9 \lambda_{\max }^{2}}{\lambda^{2}+\frac{2}{\lambda}-3 \lambda_{\max }^{2}}\right)+G_{e}\left(\lambda+\sqrt{\lambda}-\frac{1}{\lambda}-\frac{1}{\sqrt{\lambda}}\right)
$$

for the case of uniaxial tension, and

$$
\sigma_{11}^{E}-\sigma_{33}^{E}=\frac{1}{3} G_{c}\left(\lambda^{2}-\frac{1}{\lambda^{4}}\right)\left(\frac{2 \lambda^{2}+\frac{1}{\lambda^{4}}-9 \lambda_{\max }^{2}}{2 \lambda^{2}+\frac{1}{\lambda^{4}}-3 \lambda_{\max }^{2}}\right)+G_{e}\left(\lambda+\lambda^{2}-\frac{1}{\lambda}-\frac{1}{\lambda^{2}}\right)
$$

for equibiaxial tension. For the case of simple shear, the Cauchy stress is obtained through the same procedure but the eigenvalues and eigenvectors appear slightly less trivial. We obtain

$$
\sigma_{12}^{E}=\frac{1}{3} G_{c} \gamma\left(\frac{3+\gamma^{2}-9 \lambda_{\max }^{2}}{3+\gamma^{2}-3 \lambda_{\max }^{2}}\right)+\frac{G_{e}}{2}\left[\gamma\left(\lambda_{1}+\lambda_{2}\right)-\frac{\gamma^{2}}{\sqrt{4+\gamma^{2}}}\left(\lambda_{1}-\lambda_{2}\right)\right]
$$

with shear strain $\gamma$ and the two principal stretches given by $\lambda_{1}=\left(2+\gamma^{2}+\gamma \sqrt{4+\gamma^{2}}\right)^{1 / 2} / \sqrt{2}$ and $\lambda_{2}=$ $1 / \lambda_{1}$. If we solve this relationship for $\lambda_{1}$ in terms of $\gamma$, we can write $I_{1}=3+\gamma^{2}=1+\lambda_{1}^{2}+\lambda_{1}^{-2}$ for the case of simple shear. Albeit not obvious at first glance, $\sigma_{12}^{E}$ correctly reverses sign upon reversing the sign of $\gamma$. In the absence of deformation, $I_{1}=3$.

\subsection{Molecular Simulation Results on Cross-linked Network}

To test the hyperelastic models given by Arruda and Boyce (1993) and Davidson and Goulbourne (2013), we perform large scale MD simulations on the cross-linked polymer network, as listed in Tab. 2. All the MD results are reported in the reduced Lennard-Jones (LJ) units (see more details in ESI Section 3). Considering the reported energy scale is about $1000 K / k_{\mathrm{B}}$ with length scale $0.5 \mathrm{~nm}$, the corresponding stress unit is about $100 \mathrm{MPa}$ with the time unit in the picoseconds range. The different samples are carefully prepared by controlling the number of polymer chains, $n_{\mathrm{ch}}$, the number of monomers between cross-linkers, $N$, and the number of cross-linkers, $n_{\mathrm{ck}}$. In this way, we can systematically study the effects of $n_{\mathrm{ch}}, n_{\mathrm{ck}}$ and $N$ on the 
nonlinear elasticity of the cross-linked polymer network. All the samples are subjected to uniaxial tension with selected strain rates low enough, ranging from $10^{-6}$ to $10^{-5}$ (on the orders of $10^{7} \mathrm{~s}^{-1}$ in real units), that the loading and unloading curves coincide with each other. Namely, all the samples exhibit hyperelastic behavior without extra energy dissipation (viscous loss). Thus, both the Arruda-Boyce (Arruda and Boyce, 1993) and the non-affine network (Davidson and Goulbourne, 2013) models are applicable.
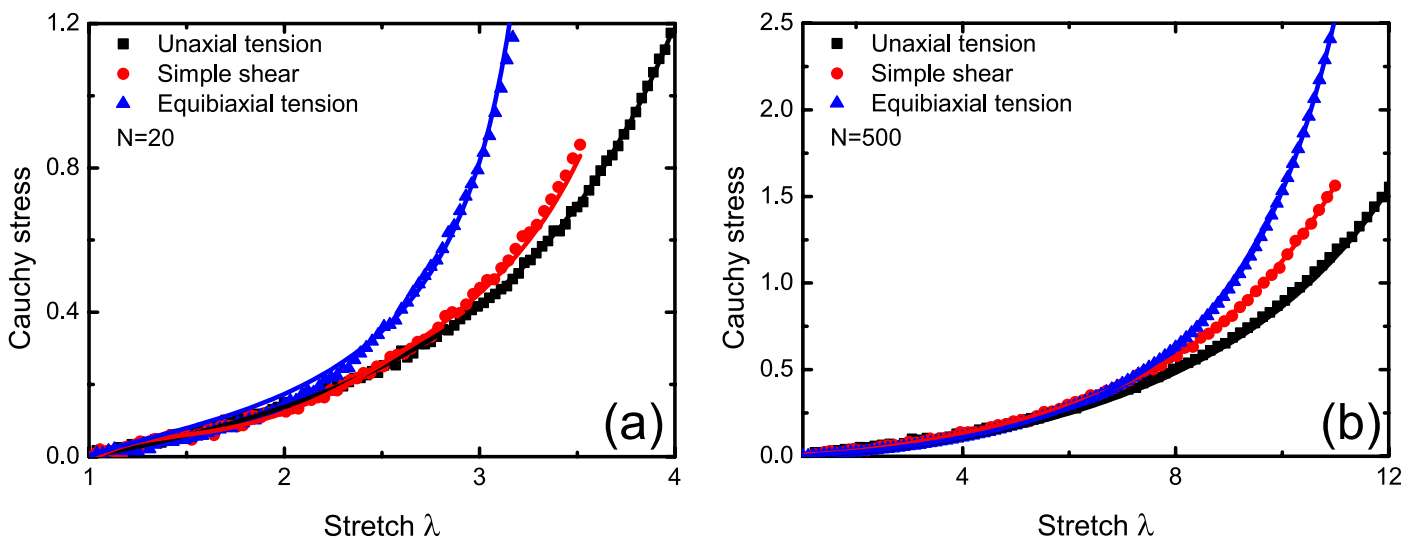

Figure 4: Molecular dynamics simulation results on cross-linked network with (a) short (unentangled) chain $N=20$ and (b) long (entangled) chain $N=500$ under uniaxial tension, simple shear and equibiaxial tension. The scattered data are given by molecular simulations, while solid lines are given by the hyperelastic continuum models.

The stress-strain curves for samples under uniaxial tension, simple shear and equibiaxial tension with $N=20$ and 500 are presented in Fig. 4. To make the stress-strain curve for simple shear comparable with that of uniaxial/equibiaxial tension, the simple shear data is plotted in terms of the maximum principal stretch $\lambda_{1}$ (diagonal direction) versus the corresponding stress (i.e. not the shear stress) along the direction corresponding to $\lambda_{1}{ }^{2}$. Therefore, the simple shear deformation is more closely related to the uniaxial tension than to equibiaxial tension as the polymer chain is being stretched along one principle direction $\left(\lambda_{1}\right)$, with chains being drawn from the transverse direction to the extension direction $\left(\lambda_{2}\right)$. In any case, the stress-strain curve for simple shear is never identical to that of uniaxial tension because it consists of pure shear and rigid body rotation at the same time. Both the Arruda-Boyce and non-affine network models are adopted to fit the uniaxial tension results given in Fig. 4. Interestingly, both of them can give excellent agreement with high fidelity (correlation coefficient $R^{2}=0.99$ ), but only if we ignore the stated microscopic expressions for their parameters. This finding alone does not automatically imply that $G_{e}$ can be considered to vanish, because $I_{1}$ is not small compared with $N$ in all cases. For example $\lambda=\lambda_{1}=4$ implies $I_{1}=16.5$ (uniaxial), 32 (equibiaxial) and 17 (shear) for $N=20$. After material parameters $\left(G_{c}\right.$ and $\lambda_{\max }$ in Arruda-Boyce model and $G_{c}, G_{e}$ and $\lambda_{\max }$ in non-affine network model) have been calibrated through the uniaxial tension, these parameters are used to predict the simple shear and equibiaxial tension by using the Arruda-Boyce or nonaffine network model. Again, the predicted stress-strain curves are found to be in good agreement with the MD simulation results (cf. Fig. 4), demonstrating that both the Arruda-Boyce and the non-affine network model are applicable for different deformation states of the cross-linked polymer network.

\footnotetext{
${ }^{2}$ The stress is obtained from the principal stresses $P_{1}$ and $P_{2}$ in the shear plane and reads $\sigma=\left(\lambda_{1} P_{1}-\lambda_{2} P_{2}\right) \sin \theta \cos \theta$ with $\theta=0.5 \arctan (2 / \gamma)\left(\right.$ Ogden, 1997). Here $\lambda_{1}=\left(2+\gamma^{2}+\gamma \sqrt{4+\gamma^{2}}\right)^{1 / 2} / \sqrt{2}$ and $\lambda_{2}=1 / \lambda_{1}$.
} 

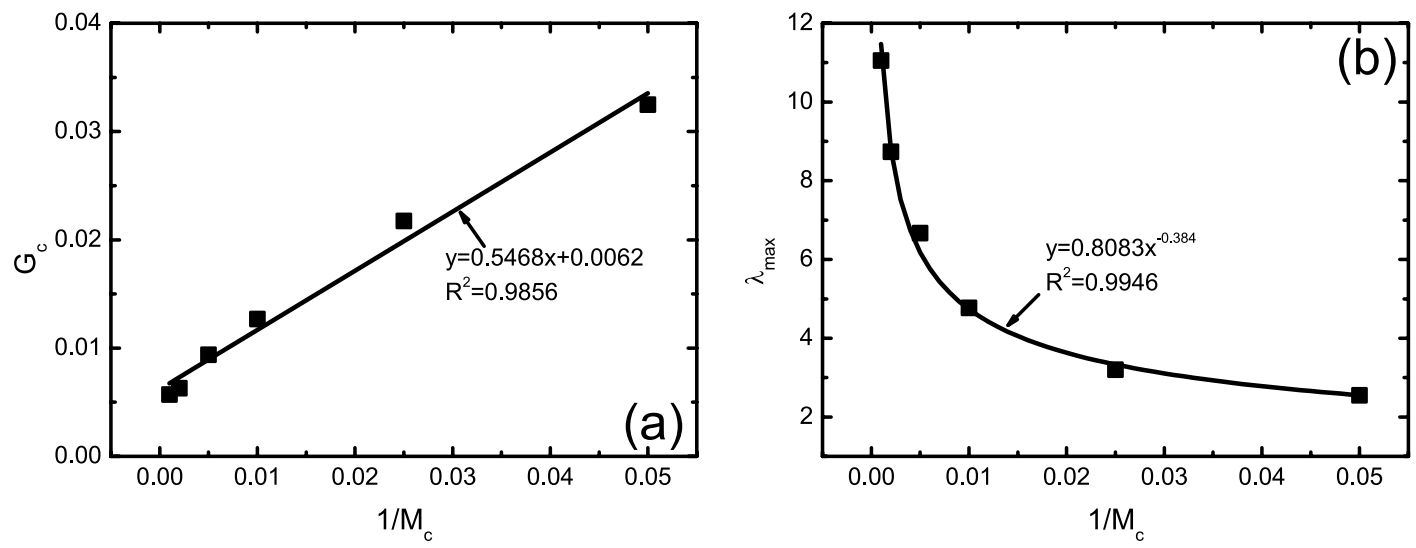

Figure 5: Calibrated material parameters (a) cross-link modulus $G_{c}$ and (b) chain extensibility $\lambda_{\max }$ in the Arruda-Boyce model. $M_{c}$ is the molecular weight of polymer segments between two cross-linkers, which is equivalent to the polymerization degree $N$.

In the Arruda-Boyce model, there are two parameters requiring determination: one is the cross-link network modulus $G_{c}$ and the other is the limited extensibility of the chain $\lambda_{\max }$. These two parameters can be calibrated through fitting the uniaxial tension data of different samples, as shown in Fig. 4. According to their definitions within this model, both of them are related to the cross-link density of the polymer network $v_{c}=\rho_{m} / M_{c}$, where $M_{c}$ is the molecular weight density of polymer segments between two cross-linkers. Assuming that $\rho_{m}$ is constant for all the samples, as they are the same material, we obtain $v_{c} \sim 1 / M_{c} \sim 1 / N$ as $M_{c}=N$. The obtained $G_{c}$ and $\lambda_{\max }$ are plotted versus $1 / M_{c}$ in Fig. 5. The fitted $G_{c}$ is linearly increasing with $1 / M_{c}$ as $G_{c}=v_{c} k_{\mathrm{B}} T=\rho_{m} k_{\mathrm{B}} T / M_{c}$ in the Arruda-Boyce model. At the same time, we find that the chain extensibility $\lambda_{\max }$ exhibits power-law behavior, $\lambda_{\max } \sim\left(1 / M_{c}\right)^{-0.384} \sim N^{0.384}$, which deviates from the expected $\lambda_{\max } \sim N^{0.5}$, signaling the non-affine deformation.

According to the non-affine network model (Davidson and Goulbourne, 2013), we need to determine the cross-link network modulus $G_{c}$, chain extensibility $\lambda_{\max }$, and the plateau modulus $G_{e}$ induced by the polymer chain entanglements. The fitted $G_{c}$ and $\lambda_{\max }$ are shown in Fig. 6. Similar to Fig. 5, $G_{c} \sim 1 / M_{c}$ and $\lambda_{\max } \sim N^{0.369}$. Moreover, the calibrated $G_{c}$ and $\lambda_{\max }$ from the non-affine network model are very close to that given by the Arruda-Boyce model.

The chain entanglement contribution, $G_{e}$, for different cross-linked polymer networks is given in Fig. 6c. When the cross-linked chain length $N$ is as small as $N=20$, there are basically no entanglements (cf. Tab. 2), and accordingly, the corresponding contribution $G_{e}$ tends to vanish, as shown in Fig. 6c. With the polymerization degree $N$ increasing, $G_{e}$ is rapidly increasing and approaches to the plateau value as $G_{e}=4 \rho_{m} k_{\mathrm{B}} T / 5 N_{e}=4 \rho_{m} k_{\mathrm{B}} T b^{2} / 5 a_{\mathrm{pp} 0}^{2} .{ }^{3}$ The tube diameters $a_{\mathrm{pp} 0}$ for the model system with different polymerization degrees $N$ are listed in Tab. 2. According to our previous study (Li et al., 2011), there exists a scaling relationship between $a_{\mathrm{pp} 0}$ and $N$ as $a_{\mathrm{pp} 0}(N)=a_{\mathrm{pp} 0}^{\infty}+a_{1} \exp \left(-N^{\chi}\right)$, where $a_{\mathrm{pp} 0}^{\infty}, a_{1}$ and $\chi$ had been obtained upon varying $N$ over a wide range. Note that $a_{\mathrm{pp} 0}^{\infty}$ represents the tube diameter for

\footnotetext{
${ }^{3}$ Here we use the tube diameter $a_{\mathrm{pp} 0}$, instead of entanglement length $N_{e}$ to estimate the plateau modulus $G_{e}$. According to the study given by Hoy et al. (2009), the entanglement length $N_{e}$ is not necessarily the appropriate quantity due to the difference between the rheological and topological entanglement lengths (cf. the different entanglement per chain values shown in Tab. 2). However, the tube diameter $a_{\mathrm{pp} 0}$ can be more accurately determined by the primitive path analysis, as demonstrated in our previous studies (Li et al., 2011, 2012b,a).
} 

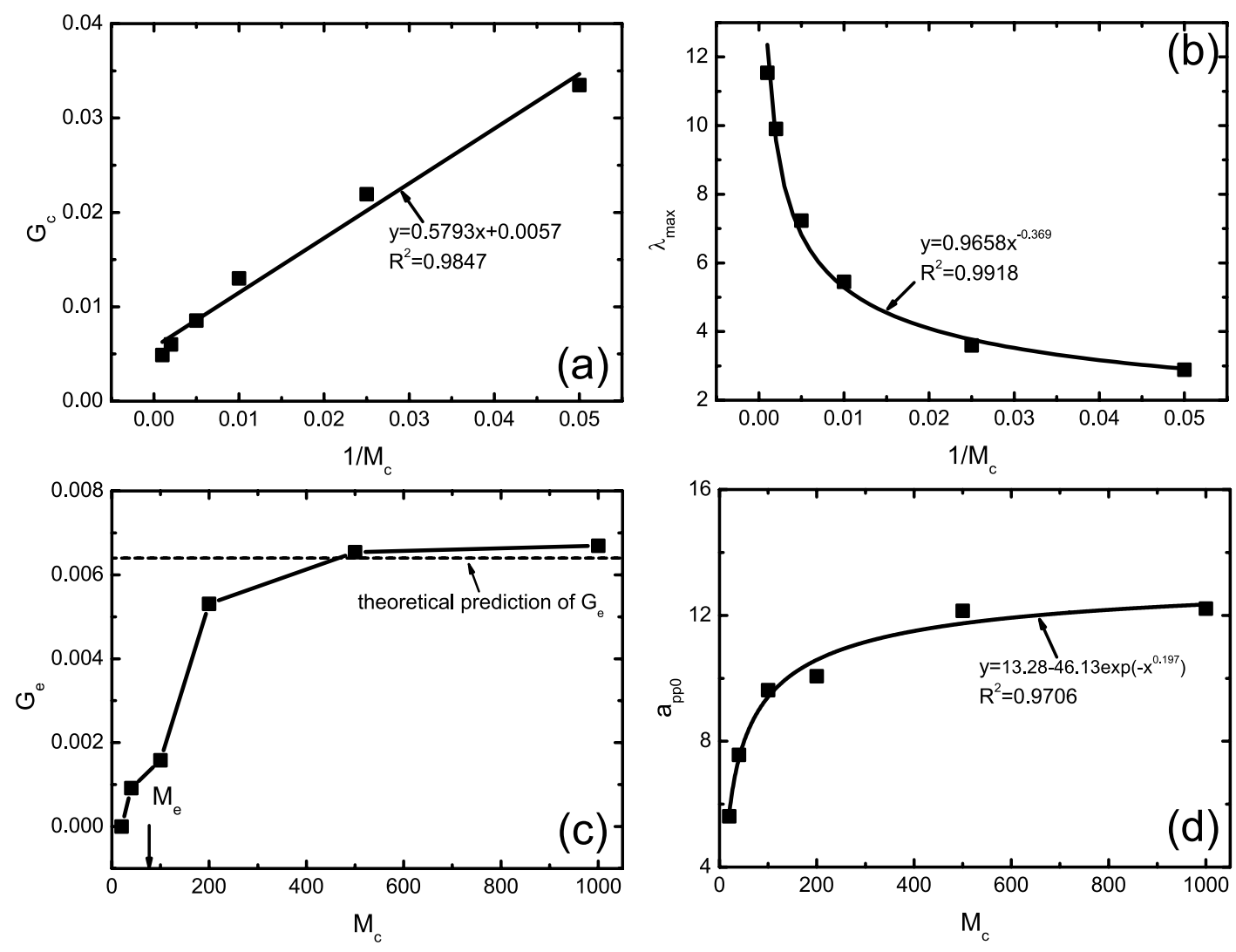

Figure 6: Calibrated material parameters (a) cross-link modulus $G_{c}$, (b) chain extensibility $\lambda_{\max }$ and (c) entanglement (plateau) modulus $G_{e}$ in the non-affine network model. (d) shows the tube diameter $a_{\mathrm{pp} 0}$ for different samples. $M_{c}$ is the molecular weight of polymer segments between two cross-linkers, which is equivalent to the polymerization degree $N$. In (c), the dashed line is the theoretical prediction by the tube model (Doi and Edwards, 1986).

infinite long chains that has to be used to determine the entanglement modulus $G_{e}$. We find $a_{\mathrm{pp} 0}^{\infty}=13.28$, $a_{1}=-46.13$ and $\chi=0.197$ (cf. Fig. 6d). This implies $G_{e}=0.0064$ for the entanglement modulus since $b^{2}=1.5706$ for the model system under study (Li et al., 2012a, 2013). More interestingly, we find that the theoretically estimated value for $G_{e}$ is very close to the fitted one, according to the uniaxial stress-strain curves (cf. Fig. 6c), when the chains are highly entangled with each other. Ultimate tests validating both the relevance of the entanglement contribution and the appropriateness of the inverse Langevin approach and its approximations require exploring the regime $I_{1}>N \gg N_{e}$ that is currently unaccessible by molecular simulation, since it requires simulation of highly entangled chains $(N \gg 1000)$ under extremely small strain rates $\left(\ll 10^{-4}\right)$.

According to above results, both the Arruda-Boyce and non-affine network models can be used to capture the stress-strain curves of cross-linked polymer networks under uniaxial tension, simple shear and equibiaxial tension with only two or three physically meaningful parameters (cf. Fig. 4). However, the Arruda-Boyce model only considers the contribution from the cross-linking, while the non-affine network model contains both cross-linking and entanglement effects. Thus, the non-affine network can simultaneously describe 

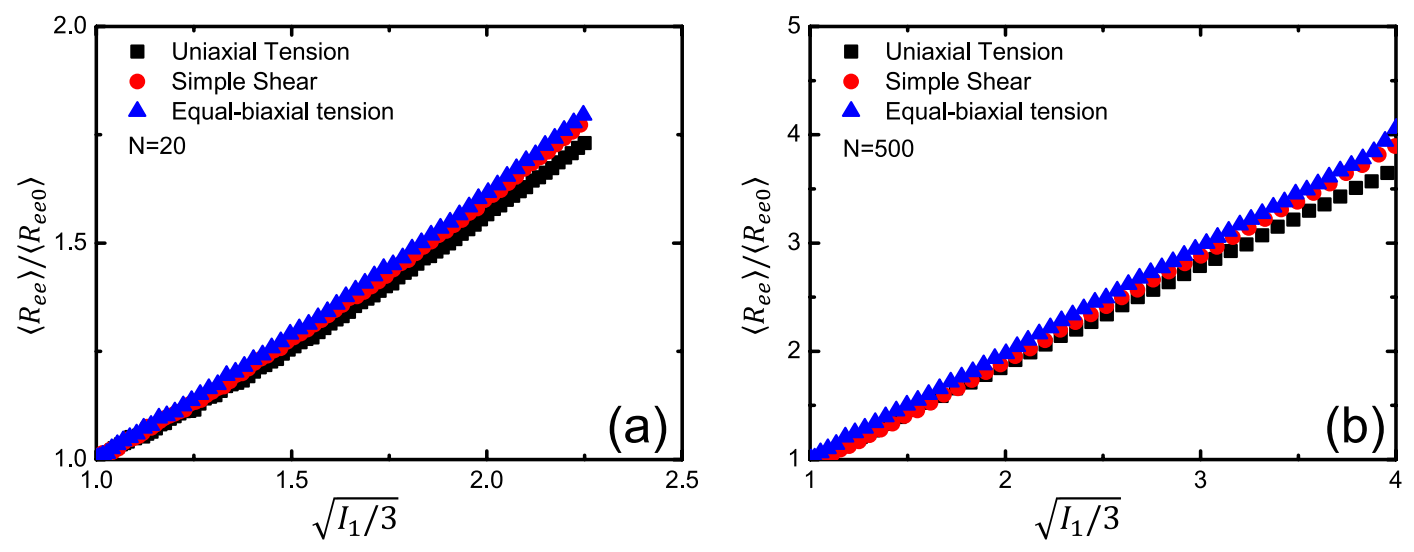

Figure 7: Normalized end-to-end distance $R_{\text {ee }}$ versus $\sqrt{I_{1} / 3}$ for cross-linked network with (a) short (unentangled) chain $N=20$ and (b) long (entangled) chain $N=500$.

the strain softening and hardening behaviors of VHB4905, which cannot be captured by the Arruda-Boyce model (cf. Fig. S2 in ESI) (Davidson and Goulbourne, 2013). Moreover, the entanglement modulus $G_{e}$ fitted from the non-affine network model is in good agreement with the theoretically estimated value, indicating that the contribution from chain entanglements is accurately represented. According to these observations, we will choose the non-affine network model to describe the hyperelastic behavior of the cross-linked polymer network.

In the above theoretical models, the chain's end-to-end distance $R_{\text {ee }}$ is assumed to be dependent on the deformation as $R_{\text {ee }} \sim \sqrt{I_{1} / 3}$ (Davidson and Goulbourne, 2013). Thus, the obtained energy density function can be applied to study different deformation states. To test this assumption, we have plotted the normalized end-to-end distance $R_{\text {ee }}$ of the polymer chain versus $\sqrt{I_{1} / 3}$, as demonstrated in Fig. 7. For different deformation states, the normalized $R_{\text {ee }}$ values collapse on a single straight line, indicating that this assumption is useful. Therefore, the calibrated parameters in the above theoretical models from uniaxial tension can be used to predict the behaviors of cross-linked polymer network under simple shear and equibiaxial tension (cf. Fig. 4).

\section{Viscous Constitutive Model for Free Chains}

\subsection{Theoretical Background}

The viscous stress originates from the diffusive behavior of free chains within the elastomer. According to the tube concept raised by Doi and Edwards (1986), the lateral movement of the free chains are constrained within a tube-like region due to the entanglements with its neighboring chains and other steric hindrances. Thus, the chain can only move back and forth, or reptate, along the centerline of the tube by Brownian motion. The centerline is the so-called "primitive chain", defined as the shortest path connecting two ends of the polymer chain upon respecting uncrossability of chains. In this way, the complex three dimensional motion of the polymer chain has been reduced into a one dimensional problem of motion with respect to the centerline. The detailed mathematical derivation is formulated based on the configuration of the primitive chain, as described in Fig. 8. Under macroscopic deformation, both the cross-linked network and free chains deform accordingly. The extension of free chains usually induces disentanglement, which is against intuition and leads to an enlarged tube diameter, thus increasing the mobility of free chains. Here we distinguish the 
chain's mobility (ability to move within the tube) and the chain's extensibility (ability to elongate under deformation).

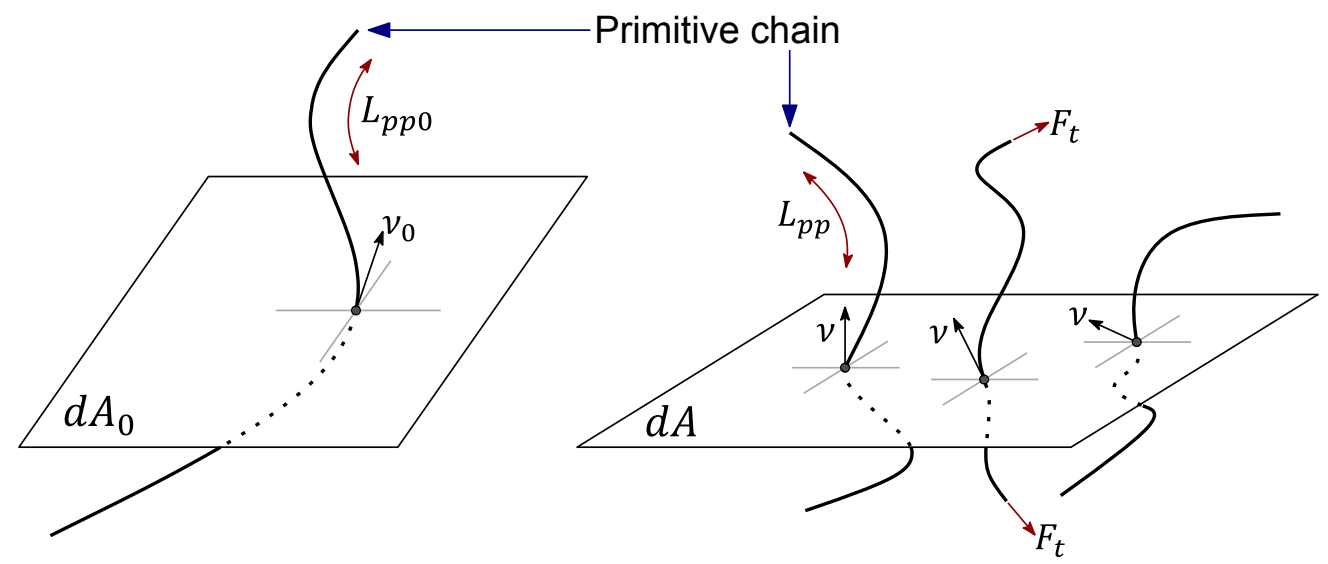

Figure 8: Primitive chain in reference (undeformed) and current (deformed) configurations. $\boldsymbol{\nu}_{0}$ and $\boldsymbol{\nu}$ are the unit tangent vectors with respect to the chain in the reference and current configurations, respectively. $F_{t}$ is the tensile force acting along the chain.

In the reference (undeformed) configuration, the unit tangent vector of the free chain at segment contour position $s$ is given by $\boldsymbol{\nu}_{0}$, as shown in Fig. 8. Similarly, the tangent vector of a chain at segment position $s$ is $\nu$ in the current (deformed) configuration. The contour lengths of the primitive chain in the reference and current configurations are denoted as $L_{\mathrm{pp} 0}$ and $L_{\mathrm{pp}}$, respectively, while $a_{\mathrm{pp} 0}$ and $a_{\mathrm{pp}}$ are the tube diameter in the initial and current configurations, respectively. With the polymerization degree of the free chains $N_{v}$, and the number of free chains per unit volume $n_{v}$, the viscous stress tensor $\sigma^{V}$ in the current configuration can be formulated according to Doi and Edwards (1986)

$$
\boldsymbol{\sigma}^{V}=n_{v}\left\langle\int_{0}^{L_{\mathrm{pp}}} F_{t} \boldsymbol{\nu} \otimes \boldsymbol{\nu} d s\right\rangle_{\boldsymbol{\nu}, L_{\mathrm{pp}}},
$$

where $F_{t}=3 k_{\mathrm{B}} T L_{\mathrm{pp}} / N_{v} b^{2}$ is the tensile force applied on the primitive chain, $\langle\star\rangle$ is the expectation operation of the quantity $\star$, which can be considered as the statistical average of the random variables $\boldsymbol{\nu}$ and $L_{\mathrm{pp}}$ over their probability space. Note that $F_{t}=3 k_{\mathrm{B}} T L_{\mathrm{pp}} / N_{v} b^{2}$ validates for $L_{\mathrm{pp}} / N_{v} b \ll 1$ (Doi and Edwards, 1986), which is true for highly entangled chains. For example, when $N_{v}=1000$, the corresponding primitive chain length $L_{\mathrm{pp}}=127$ with Kuhn length $b=1.25$ (cf. Tab. 2), thus $L_{\mathrm{pp}} / N_{v} b \ll 1$. Under the affine deformation assumption, the unit tangent vector of the free chain is oriented according to the macroscopic deformation, $\boldsymbol{\nu}=\mathbf{F} \cdot \boldsymbol{\nu}_{0} /\left\|\mathbf{F} \cdot \boldsymbol{\nu}_{0}\right\|$. Note that these tangents are to be assumed affine. One further considers the deformation gradient $\mathbf{F}$ to be constant over the spatial extension of a single free chain and thus insensitive to segment position $s$. A single segment orientational probability distribution function (PDF) $f(\boldsymbol{\nu}, s, t)$ can then be formulated for the tangent vector $\boldsymbol{\nu}$ at the primitive chain segment position $s$ and time $t$. This PDF must be isotropic in the reference configuration at time $t_{0}$, i.e. $f\left(\boldsymbol{\nu}_{0}, s, t=t_{0}\right)=1 / 4 \pi$, reflecting a random orientation of tangent vectors. The form of the PDF at a later time $t$ can be obtained through Boltzmann's superposition principle from information at intermediate times $t^{\prime} \in[0, t]$ (Doi and 
Edwards, 1986)

$$
f(\boldsymbol{\nu}, s, t)=\int_{0}^{t} \frac{\partial \Psi\left(s, t-t^{\prime}\right)}{\partial t^{\prime}} d t^{\prime} \int_{S^{2}} \frac{1}{4 \pi} \delta\left(\boldsymbol{\nu}-\frac{\mathbf{F}\left(t, t^{\prime}\right) \cdot \boldsymbol{\nu}_{0}}{\left\|\mathbf{F}\left(t, t^{\prime}\right) \cdot \boldsymbol{\nu}_{0}\right\|}\right) d^{2} \nu_{0},
$$

where $\Psi(s, \Delta t)$ stands for the survival probability function characterizing the probability of segment $s$ to remain within its tube for duration $\Delta t . \mathbf{F}\left(t, t^{\prime}\right)$ is the time-dependent deformation gradient. $\int_{S^{2}}$ and $\delta(\star)$ denote an integration over the unit sphere and the Dirac- $\delta$ distribution, respectively. Following Doi and Edwards (1986)

$$
\Psi(s, t)=\sum_{p=1, \text { odd }}^{\infty} \frac{4}{p \pi} \sin \left(\frac{p \pi s}{L_{\mathrm{pp}}}\right) \exp \left(-\frac{p^{2} t}{\tau_{d}}\right),
$$

where $\tau_{d}$ is the disentanglement time for the free chain, representing the relaxation behavior of chains. $\Psi(s, t)$ can be determined from the microscopic diffusion equation for free chains (Doi and Edwards, 1986), and the viscous Cauchy stress can be rewritten as

$$
\boldsymbol{\sigma}^{V}(t)=n_{v}\left\langle\int_{S^{2}} \int_{0}^{L_{\mathrm{pp}}} F_{t} \boldsymbol{\nu} \otimes \boldsymbol{\nu} f(\boldsymbol{\nu}, s, t) d s d^{2} \nu_{0}\right\rangle_{L_{\mathrm{pp}}},
$$

where the expectation has been considered with respect to the tangent vector $\nu$ through the PDF $f$, and the only remaining random variable is the primitive chain length $L_{\mathrm{pp}}$. The integral over the unit sphere will be be approximated by a discrete sum in our finite element analysis.

Miehe and Göktepe (2005) assumed that the viscous behavior was governed by a spectrum of $a=1 \ldots \Xi$ prototype chains superimposed onto the elastic chain network (it can be thought as chains on a microsphere). For each chain, the viscous deformation is described by the phenomenological forms given by the classical viscoelasticity

$$
\dot{\beta}_{y}^{a}+\frac{1}{\tau_{y}^{a}}\left|\beta_{y}^{a}\right|^{\delta_{y}^{a}-1} \beta_{y}^{a}=\mu_{y}^{a} \frac{d}{d t}\left(\ln \lambda_{y}\right),
$$

where $\beta_{y}^{a}, \mu_{y}^{a}$ and $\tau_{y}^{a}$ are the microforce on the chain, overstress moduli and relaxation time, respectively. $\lambda_{y}$ is the relative stretch of a chain. The superscript $a=1 \ldots \Xi$ denotes the different chains. The average microforce on a microsphere determines the viscous stress response. However, in the present work, the viscous behavior of a single chain is governed by the reptation model, derived based on the tube survival probability function. The microsphere model is only used to perform the integral of Eq. (14) over the tangent vector of the chain (other integral methods can also be applied). Evidently, our model given in Eq. (14) can dramatically reduce the number of material parameters, as the phenomenological model in Miehe and Göktepe requires $6 \Xi$ parameters for the viscous part (Miehe and Göktepe, 2005).

By plugging Eq. (12) and Eq. (13) into Eq. (14), the viscous Cauchy stress can be reformulated as

$$
\boldsymbol{\sigma}^{V}(t)=G\left\langle L_{\mathrm{pp}}\right\rangle^{2} \sum_{p=1, \text { odd }}^{\infty} \int_{0}^{t} \boldsymbol{\Upsilon}\left(p, t^{\prime}, t, \tau_{d}\right) d t^{\prime},
$$

with $G=3 k_{\mathrm{B}} T n_{v} / N_{v} b^{2}$ and the integration kernel function $\Upsilon\left(p, t^{\prime}, t, \tau_{d}\right)=\mathscr{E}\left(p, t^{\prime}, t, \tau_{d}\right) \boldsymbol{\Lambda}\left(t, t^{\prime}\right)$ that is a product of a mode-dependent scalar

$$
\mathscr{E}\left(p, t^{\prime}, t, \tau_{d}\right)=\frac{8}{\pi^{2} \tau_{d}} \exp \left(p^{2} \frac{t-t^{\prime}}{\tau_{d}}\right),
$$


and matrix

$$
\boldsymbol{\Lambda}\left(t, t^{\prime}\right)=\int_{S^{2}} \boldsymbol{\nu} \otimes \boldsymbol{\nu} d^{2} \nu \int_{S^{2}} \frac{1}{4 \pi} \delta\left(\boldsymbol{\nu}-\frac{\mathbf{F}\left(t, t^{\prime}\right) \cdot \boldsymbol{\nu}_{0}}{\left\|\mathbf{F}\left(t, t^{\prime}\right) \cdot \boldsymbol{\nu}_{0}\right\|}\right) d^{2} \nu_{0}-\frac{1}{3} \mathbf{1} .
$$

Here, $\mathbf{1}$ is the 2 nd rank identity tensor.

The above formulations have been found to work well in describing the viscous behavior of polymer chains under small deformation ( $\mathrm{Li}$ et al., 2012b). However, under the finite strain deformation it cannot be used to capture the viscous energy dissipation of chains, since several important physical mechanisms have been neglected in the original formulation ( $\mathrm{Li}$ et al., 2012b) ; more specifically, primitive chain length and tube diameter should be changed by the deformation gradient, which has already been observed through experiments (Straube et al., 1995; Pyckhout-Hintzen et al., 2013; Ott et al., 2014). Therefore, we have made the following important assumptions for these physical mechanisms

1. Change of primitive chain length $L_{\mathrm{pp}}$. The viscous stress contributed by the free chains relies on the primitive chain length $\left\langle L_{\mathrm{pp}}\right\rangle$, as shown in Eq. (15), which has been averaged over all the possible initial tangent vectors $\boldsymbol{\nu}_{0}$ for these chains. In the reference configuration, the distribution of $\boldsymbol{\nu}_{0}$ is isotropic and the primitive chain length $L_{\mathrm{pp}}=L_{\mathrm{pp} 0}\left\|\mathbf{F} \cdot \boldsymbol{\nu}_{0}\right\|$, therefore the contribution of the change in primitive chain length can be formulated as

$$
\left\langle L_{\mathrm{pp}}\right\rangle=\frac{L_{\mathrm{pp} 0}}{4 \pi} \int_{S^{2}}\left\|\mathbf{F} \cdot \boldsymbol{\nu}_{0}\right\| d^{2} \nu_{0}
$$

The above equation predicts a unique dependency of $\left\langle L_{\mathrm{pp}}\right\rangle$ on time for a given deformation state. We should emphasize that such a relationship between the primitive chain length $\left\langle L_{\mathrm{pp}}\right\rangle$ and deformation gradient $\mathbf{F}$ can be verified through our large scale molecular simulations and primitive path analysis, which has been demonstrated before for the entangled polymer network under uniaxial tension (Li et al., 2012b, 2013). Because $L_{\mathrm{pp}}$ cannot exceed the contour length of the chain (otherwise the polymer chain will be broken), the applicability of Eq. (18) is limited to this regime.

2. Change of tube diameter $a_{\mathrm{pp}}$. The viscous stress depends on the disentanglement time $\tau_{d}$, which is a function of tube diameter $a_{\mathrm{pp}}$ as (Doi and Edwards, 1986)

$$
\tau_{d}=\frac{\xi n_{v}^{3} b^{4}}{\pi^{2} k_{\mathrm{B}} T\left\langle a_{\mathrm{pp}}\right\rangle^{2}}=\frac{L_{\mathrm{pp}}^{2}}{\pi^{2} D_{c}},
$$

where $\xi$ is the monomer friction coefficient and $D_{c}$ is the diffusion coefficient. We write $\left\langle a_{\mathrm{pp}}\right\rangle$ instead of $a_{\mathrm{pp}}$ because we are going to consider a deformation-dependent $\left\langle a_{\mathrm{pp}}\right\rangle$ and $\tau_{d}$. When the contour length of the primitive chain extends, the neighboring chains elongate and they are gradually disentangled, resulting in a lower level of geometric constraint on the deforming tube with enlarged tube diameter $a_{\mathrm{pp}}$. On the contrary, the tube diameter $a_{\mathrm{pp}}$ will be reduced with higher levels of geometric constraints due to the compactness of chains. The tube diameter $a_{\mathrm{pp}}$ is related to the end-to-end distance $R_{\mathrm{ee}}$ and primitive chain length $L_{\mathrm{pp}}$ as $a_{\mathrm{pp}}=\left\langle R_{\mathrm{ee}}^{2}\right\rangle / L_{\mathrm{pp}}$ (Doi and Edwards, 1986), which is found by assuming the primitive path of the polymer chain is a random walk with constant step $a_{\mathrm{pp}}$. The probability distribution function for the end-to-end vector $\mathbf{R}_{\mathrm{ee}}$ in equilibrium is given by $f_{0}\left(\mathbf{R}_{\mathrm{ee}}\right)$ with

$$
f_{0}(\mathbf{R})=\left(\frac{3}{2 \pi R_{\mathrm{ee} 0}^{2}}\right)^{3 / 2} \exp \left(-\frac{3 R^{2}}{2 R_{\mathrm{ee} 0}^{2}}\right), \quad R_{\mathrm{ee} 0}^{2}=N_{v} b^{2}
$$

Thus, the mean squared end-to-end distance $R_{\mathrm{ee}}$ under the deformation gradient $\mathbf{F}$ can be calculated as

$$
\left\langle R_{\mathrm{ee}}^{2}\right\rangle=\int\|\mathbf{F} \cdot \mathbf{R}\|^{2} f_{0}(\mathbf{R}) d^{3} R .
$$


The change of mean tube diameter $a_{\mathrm{pp}}$ can be quantified with an affine deformation assumption as

$$
\left\langle a_{\mathrm{pp}}\right\rangle=\frac{\left\langle R_{\mathrm{ee}}^{2}\right\rangle}{\left\langle L_{\mathrm{pp}}\right\rangle}=\frac{4 \pi \int\|\mathbf{F} \cdot \mathbf{R}\|^{2} f_{0}(\mathbf{R}) d^{3} R}{L_{\mathrm{pp}}^{0} \int\left\|\mathbf{F} \cdot \boldsymbol{\nu}_{0}\right\| d^{2} \nu_{0}} .
$$

According to the changes of mean tube diameter $\left\langle a_{\mathrm{pp}}\right\rangle$, the variation of the disentanglement time $\tau_{d}$ can be easily estimated by the Eq. (19) We are going to denote the tube diameter and disentanglement time as $a_{\mathrm{pp} 0}$ and $\tau_{d 0}$ in the absence of deformation, respectively.

3. Change of chain orientation. The viscous stress arising from free chains also depends on the quantity $\boldsymbol{\Lambda}\left(t, t^{\prime}\right)$ through the integration kernel $\boldsymbol{\Upsilon}$, as shown in Eq. (15). Here $\boldsymbol{\Lambda}\left(t, t^{\prime}\right)$ determines the rotation of the primitive chains. Intuitively, the larger the rotation of chains, the larger viscous stress response in the material. The continuous form of $\boldsymbol{\Lambda}\left(t, t^{\prime}\right)$ is given in Eq. (17).

4. Fractional order viscoelasticity. The viscoelastic behaviors of polymer chains can be modeled based on fractional calculus. The governing equations for these materials are given by fractional differential equations. A spring and a dashpot can be used to represent a zero-order element (purely elastic response) and a first-order element (purely viscous response), respectively. Thus, a fractional order $0<\alpha<1$ results in a new element in order to interpolate between the purely elastic and viscous behaviors, which is the so-called spring-pot. The fractional element introduces an interesting physical interpretation, for example, a fractal tree-like hierarchical structure, allowing for separation of the material and global time scales. Comparing with the discrete relaxation spectrum of the linear Voigt model, the fractional-order model exhibits a continuous relaxation distribution (Schiessel et al., 1995; Craiem et al., 2008). To consider the polydispersity of the chain's molecular weight and the cross-linking effect on the diffusion of free chains, the generalized Mittag-Leffler function $E_{\alpha, 1}(x)=\sum_{k=0}^{\infty} x^{k} /(\alpha k)$ ! has been introduced into the probability function $\Psi(s, t)$ as (Tang et al., 2012; Li et al., 2012b)

$$
\Psi(s, t)=\sum_{p=1, \text { odd }}^{\infty} \frac{4}{p \pi} \sin \left(\frac{p \pi s}{L_{p p}}\right) E_{\alpha, 1}\left[-p^{2}\left(\frac{t}{\tau_{d}}\right)^{\alpha}\right],
$$

with a new parameter $\alpha$ accounting for the effect of cross-linking or nonuniform distribution of the molecular weight on the disentanglement time $\tau_{d}$. For $\alpha=1$ the above equation reduces to the standard Doi-Edwards model (Doi and Edwards, 1986), as $E_{1,1}(x)=\exp (x)$. The introduction of $\alpha$ can improve the prediction of the relaxation behavior of polymer melts with high polydispersity, as demonstrated in our previous study (Li et al., 2012b). The fractional order viscoelasticity of polymers and soft biological tissues has also been observed through experiments (Meral et al., 2010; Müller et al., 2011).

With the above important modifications at hand, we can reformulate the original Doi-Edwards model Eq. (15) to study the finite strain viscoelastic behavior of an elastomer. The newly formulated viscous stress $\boldsymbol{\sigma}^{V}$ is again written in the form of Eq. (15) with the unchanged expression for $\boldsymbol{\Lambda}\left(t, t^{\prime}\right)$ given by Eq. (17), while we use the newly proposed deformation-dependent expressions for $\left\langle L_{\mathrm{pp}}\right\rangle$ and $\tau_{d}$ given by Eqs. (18), (22), and (19), as well as a modified expression for the scalar part of the kernel function. That is, Eq. (16) is replaced by

$$
\mathscr{E}\left(p, t^{\prime}, t, \tau_{d}\right)=\frac{8}{\pi^{2} \tau_{d}}\left[-\frac{\partial}{\partial x} E_{\alpha, 1}\left(-p^{2} x^{\alpha}\right)\right]_{x=\left(t-t^{\prime}\right) / \tau_{d}},
$$

and thus original Doi-Edwards tube model recovered exactly for $\alpha=1$. 
Note that in the above formulation, the changes of primitive chain length $L_{\mathrm{pp}}$, tube diameter $a_{\mathrm{pp}}$ and the rotation of free chains $\nu$ require the evaluation of integrals over their continuous space. To fulfill this requirement within finite element analysis, the microsphere model is adopted by discretizing the continuous orientation distribution of the unit sphere $S^{2}$ by $K$ discrete orientation vectors $\left\{\boldsymbol{\nu}_{k}\right\}_{k=1, \ldots, K}$ with corresponding weight factors $\left\{w_{k}\right\}_{k=1, \ldots, K}$. Such a method is suitable for our case as all the components of the viscous stress are determined by these reference vectors. Miehe and Göktepe (2005) have given a suitable set of orientation vectors $\left\{\boldsymbol{\nu}_{k}\right\}$ and weight factors $\left\{w_{k}\right\}$ for $K=21$. According to these sets, the continuous integrals given in Eq. (18) and Eq. (15) can be reformulated as discrete sums. Moreover, the viscous Cauchy stress is expressed in terms of first Piola-Kirchhoff stress as $J \sigma^{V}=\mathbf{P}^{V} \cdot \mathbf{F}^{T}$ in the numerical simulations. Such a procedure is similar to Gauss-quadrature and transforms the continuous variables over the initial tangent vectors into a discrete sum, as given below

$$
\frac{1}{4 \pi} \int_{S^{2}} \Theta\left(\boldsymbol{\nu}_{0}\right) d \boldsymbol{\nu}_{0}^{2}=\sum_{k=1}^{K} \Theta_{k}\left(\boldsymbol{\nu}_{0(k)}\right) w_{k}
$$

where $\Theta$ is an arbitrary function.

Applying Eq. (25) and Eq. (24) to Eqs. (15)-(17), the viscous stress can be expressed in the following discrete version

$$
\begin{aligned}
\boldsymbol{\sigma}^{V}(t)= & \frac{3 k_{\mathrm{B}} T n_{v}}{N_{v} b^{2}}\left\langle L_{\mathrm{pp}}\right\rangle^{2} \sum_{p=1, \mathrm{odd}}^{\infty} \int_{0}^{t} \frac{8}{\pi^{2} \tau_{d}}\left[-\frac{\partial}{\partial x} E_{\alpha, 1}\left(-p^{2} x^{\alpha}\right)\right]_{x=\left(t-t^{\prime}\right) / \tau_{d}} \\
& \times\left\{\sum_{k=1}^{K} \frac{\left[\mathbf{F}\left(t, t^{\prime}\right) \cdot \nu_{0(k)}\right] \otimes\left[\mathbf{F}\left(t, t^{\prime}\right) \cdot \nu_{0(k)}\right]}{\left\|\mathbf{F}\left(t, t^{\prime}\right) \cdot \nu_{0}(k)\right\|^{2}} w_{k}-\frac{1}{3} \mathbf{1}\right\} d t^{\prime}
\end{aligned}
$$

with the primitive chain length $\left\langle L_{\mathrm{pp}}\right\rangle^{2}=L_{\mathrm{pp} 0}^{2} \sum_{k=1}^{K}\left\|\mathbf{F} \cdot \nu_{0(k)}\right\|^{2} w_{k}$. Note that $\tau_{d}$ depends on the tube diameter $a_{\mathrm{pp}}$ and tangent vector $\boldsymbol{\nu}_{0}$ through Eqs. (19)-(22).

\subsection{Molecular Simulation Results on Free Chains}

In the above theoretical formulation, we have made several important assumptions regarding the changes of the primitive chain length $L_{\mathrm{pp}}$ and tube diameter $a_{\mathrm{pp}}$. To further verify these assumptions, we have performed large scale MD simulations on highly entangled polymers and have measured $\left\langle L_{\mathrm{pp}}\right\rangle$ and $\left\langle a_{\mathrm{pp}}\right\rangle$ directly. To simplify the comparison, we begin by evaluating $\left\langle L_{\mathrm{pp}}\right\rangle$ and $\left\langle a_{\mathrm{pp}}\right\rangle$ for uniaxial tension using the theoretical expressions. In that case the deformation gradient tensor reads

$$
\mathbf{F}=\left(\begin{array}{ccc}
1 / \sqrt{\lambda} & 0 & 0 \\
0 & 1 / \sqrt{\lambda} & 0 \\
0 & 0 & \lambda
\end{array}\right)
$$

where $\lambda$ is the stretch ratio along the tensile direction. The unit tangent vector $\nu_{0}$ of the primitive chain in the reference configuration can be written as

$$
\boldsymbol{\nu}_{0}=\left(\begin{array}{c}
\sqrt{1-z^{2}} \cos \varphi \\
\sqrt{1-z^{2}} \sin \varphi \\
z
\end{array}\right)
$$



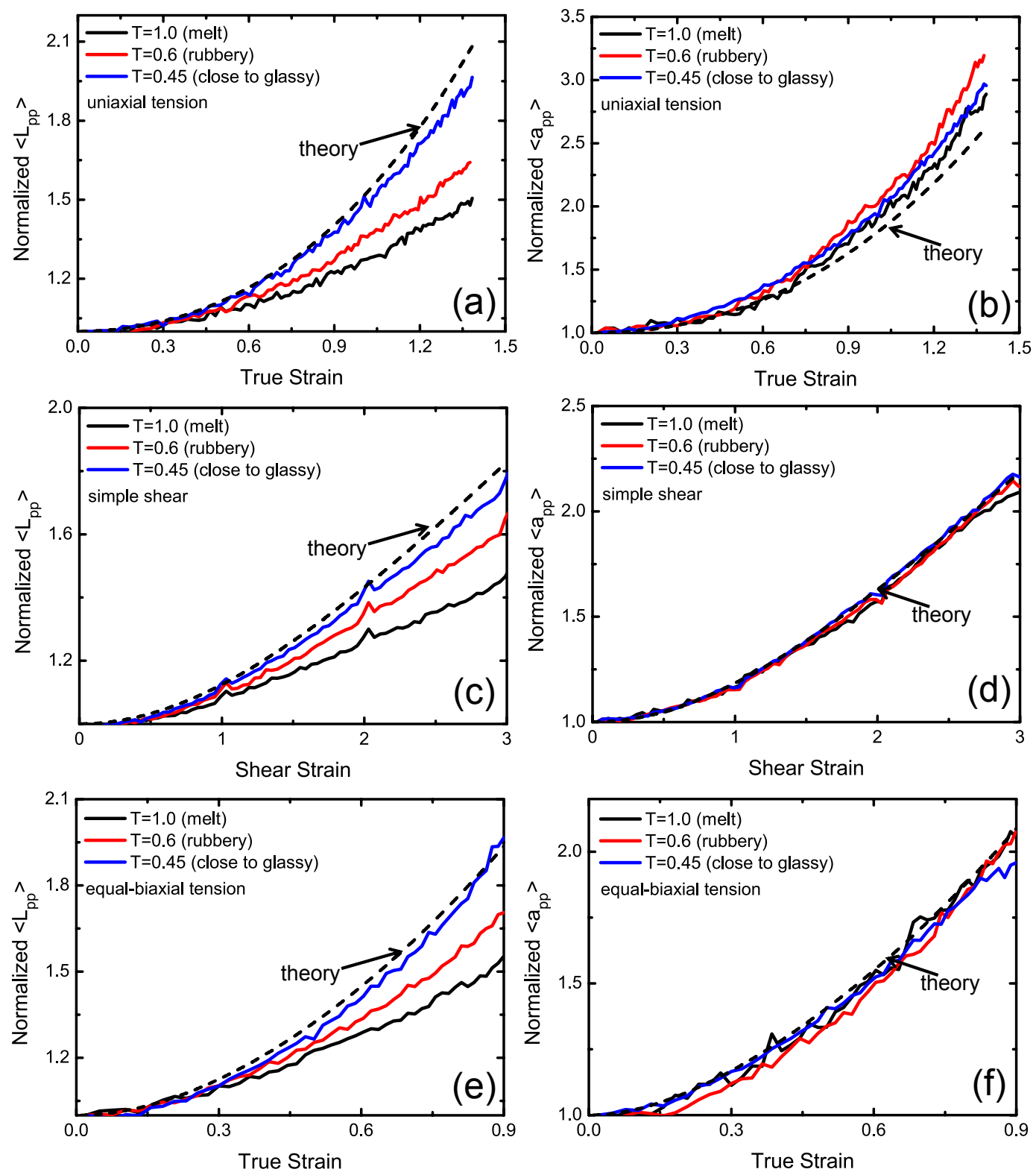

Figure 9: Primitive path analysis results on changes of contour length of the primitive chain $\left\langle L_{\mathrm{pp}}\right\rangle / L_{\mathrm{pp} 0}$ (left) and tube diameter $\left\langle a_{\mathrm{pp}}\right\rangle / a_{\mathrm{pp} 0}$ (right column) during (a)-(b) uniaxial tension, (c)-(d) simple shear and (e)-(f) equal-biaxial tension vs true strain $\epsilon=$ $\exp (\lambda)$ and shear strain $\gamma$. The dashed lines are given by our theoretical formulation based on affine deformation assumption (cf. Eqs. 18 and 22), explicitly stated for the case of uniaxial deformation in Eqs. 29 and 31. The polymerization degree of the model system is $N=500$ with 200 chains. For the case of simple shear, each dimension of the simulation box has been enlarged twice within the shear plane. The loading rate of molecular simulations is $10^{-5}$ (reduced LJ unit), which is the slowest loading rate achievable due to the limitation of computational facility. The true strain is defined as $\ln (\lambda)$, which is the usual expression for Hencky true strain. 
where $\varphi \in[0,2 \pi]$ and $z \in[-1,1]$. Therefore, according to our above assumption (cf. Eq. 18), the contour length of the primitive chain during uniaxial deformation can be calculated analytically

$$
\frac{\left\langle L_{\mathrm{pp}}\right\rangle(\lambda)}{L_{\mathrm{pp} 0}}=\frac{1}{4 \pi} \int_{0}^{2 \pi} \int_{-1}^{1}\left\|\mathbf{F} \cdot \boldsymbol{\nu}_{0}\right\| d z d \varphi=\frac{1}{2}\left(\lambda+\frac{\sinh ^{-1} \sqrt{\lambda^{3}-1}}{\sqrt{\lambda^{4}-\lambda}}\right),
$$

where $\sinh ^{-1}$ is the inverse sinh. Similarly, the mean squared end-to-end distance, $\left\langle R_{\mathrm{ee}}^{2}\right\rangle$, in the deformed state evaluates to

$$
\frac{\left\langle R_{\mathrm{ee}}^{2}\right\rangle(\lambda)}{\left\langle R_{\mathrm{ee}}^{2}\right\rangle_{0}}=\frac{1}{\left\langle R_{\mathrm{ee}}^{2}\right\rangle_{0}} \int(\mathbf{F} \cdot \mathbf{R})^{2} f_{0}(\mathbf{R}) d^{3} R=\frac{2+\lambda^{3}}{3 \lambda}
$$

and the corresponding change of the mean tube diameter, $\left\langle a_{\mathrm{pp}}\right\rangle$, is

$$
\frac{\left\langle a_{\mathrm{pp}}\right\rangle(\lambda)}{a_{\mathrm{pp} 0}}=\frac{\left\langle R_{\mathrm{ee}}^{2}\right\rangle(\lambda)}{\left\langle L_{\mathrm{pp}}\right\rangle(\lambda)} \frac{L_{\mathrm{pp} 0}}{\left\langle R_{\mathrm{ee}}^{2}\right\rangle_{0}}=\frac{2\left(2+\lambda^{3}\right)}{3 \lambda\left(\lambda+\frac{\sinh ^{-1} \sqrt{\lambda^{3}-1}}{\sqrt{\lambda^{4}-\lambda}}\right)}
$$

Similar relationships can be derived for simple shear and equibiaxial tension. The above derived Eqs. (29)(31) are compared with molecular simulation results in Fig. 9.

As presented in Fig. 9, the molecular dynamics simulation results on changes of primitive chain length $\left\langle L_{\mathrm{pp}}\right\rangle$ and tube diameter $\left\langle a_{\mathrm{pp}}\right\rangle$ under uniaxial tension, simple shear and equibiaxial tension are compared with our above theoretical formulations. Interestingly, the change of $\left\langle a_{\mathrm{pp}}\right\rangle$ is in good agreement with our theoretical formulation, regardless of the deformation state or temperature. The change of $\left\langle L_{\mathrm{pp}}\right\rangle$ demonstrates different behaviors. When the temperature is very high, for example, the polymer is in a molten state, the variation of $\left\langle L_{\mathrm{pp}}\right\rangle$ stays well below the affine prediction given by our above formulations. With decreasing temperature, $\left\langle L_{\mathrm{pp}}\right\rangle$ has been enlarged at the same amount of applied strain. Eventually, when the polymer is close to its glassy state, $\left\langle L_{\mathrm{pp}}\right\rangle$ behaves as prescribed by our affine deformation assumption (Eq. 29).

Here we may wonder how these interesting phenomena happened. In an attempt to answer this question, we make the analogy of the polymer system to a fluid or solid, according to different temperatures. When the temperature is very high, for example, $T=1.0$, the polymer is in the melt state, mimicking the behavior of fluid. Thus, when the chains are under external deformation, large fluctuations accompany the deformation of these chains. Since the number of entanglements per chain is still quite small (about 10 in this case) ${ }^{4}$, the chain can easily slide during the deformation at this high temperature. Therefore, the affine deformation assumption is not applicable in this case. When the temperature of the system is reduced sufficiently close to the glass transition temperature $T_{g}$, the polymer is more close to its solid state. During the deformation, the thermal activation energy is rather high. The chain is constrained by the entanglements and cannot slide. Therefore, the change of $\left\langle L_{\mathrm{pp}}\right\rangle$ is very well described by the affine deformation assumption, as given in Fig. 9. Here we should also emphasize that although the change of $\left\langle a_{\mathrm{pp}}\right\rangle$ is very close to our theoretical prediction at all studied temperatures, it does not imply that the underlying affine deformation assumption holds. Because $\left\langle a_{\mathrm{pp}}\right\rangle$ is a ratio between two quantities $\left\langle R_{\mathrm{ee}}^{2}\right\rangle$ and $\left\langle L_{\mathrm{pp}}\right\rangle$ that demonstrate nonaffine deformation behaviors at high temperature, these effects are actually seen to cancel each other out. Therefore, $\left\langle a_{\mathrm{pp}}\right\rangle$ changes according to the affine deformation assumptions at different temperatures in our simulations.

${ }^{4}$ The average molecular weight between two neighboring entanglements is about $3120 \mathrm{~g} / \mathrm{mol}$ for cis-1,4 polyisoprene (natural rubber) at room temperature (Toki et al., 2013). Therefore, a typical polymer chain of natural rubber has more than 300 entanglements, considering its molecular weight is around $10^{6} \mathrm{~g} / \mathrm{mol}$. 


\section{Numerical Examples}
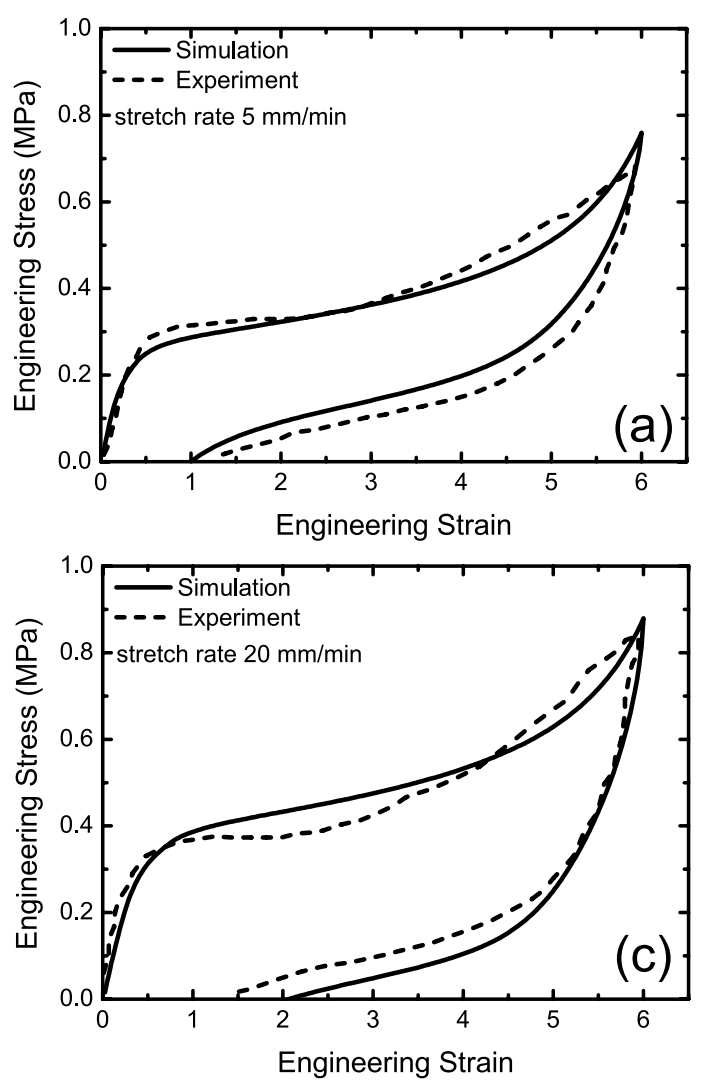
strain viscoelasticity of elastomers.
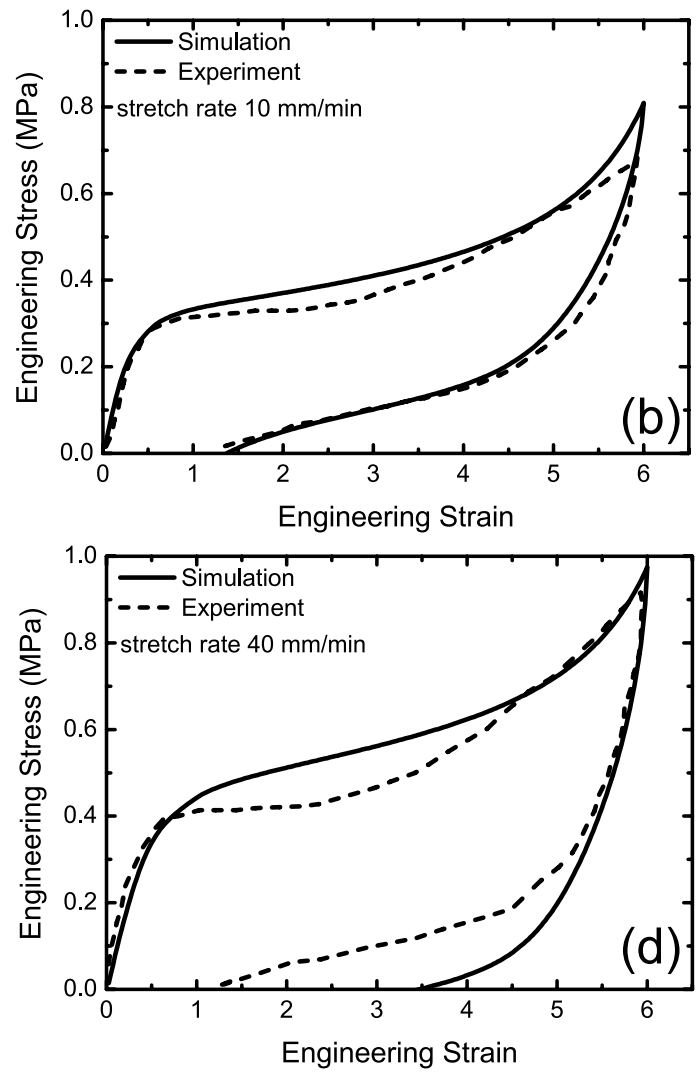

Figure 10: Effect of stretch speed on uniaxial loading and unloading behaviors of un-vulcanized NR at room temperature: (a) $5 \mathrm{~mm} / \mathrm{min}$, (b) $10 \mathrm{~mm} / \mathrm{min}$, (c) $20 \mathrm{~mm} / \mathrm{min}$ and (d) $40 \mathrm{~mm} / \mathrm{min}$, corresponding to strain rates 0.006 to $0.05 \mathrm{~s}^{-1}$. The experimental results are taken from Amnuaypornsri et al. (2012) and plotted versus the engineering strain $\lambda-1$. The material parameters of our constitutive model are collected in Tab. 3 .

Since the disentanglement time $\tau_{d}$ is mainly affected by the tube diameter, cf. Eq. (19), we have essentially provided an argument in favor of using the affine deformation assumption in our constitutive model for finite

By combining the hyperelastic (non-affine network) and viscous (refined tube) continuum models discussed in the above two sections, a complete viscoelastic constitutive model has been developed for which all its parameters have direct physical meaning. The stress tensor is thus calculated by adding elastic $\sigma^{E}$ and viscous $\sigma^{V}$ contributions using Eqs. (15), (17)-(19), (22) and (24) to calculate $\sigma^{V}$, with $W=W^{\text {nonaffine }}$ from Eq. (7) to calculate $\sigma^{E}$. The developed constitutive model has been implemented into the commercial finite element software, ABAQUS, for finite element analysis. The implementation of the viscous stress has been given in our previous study (Tang et al., 2012), and the finite element implementation of the non-affine network model for hyperelasticity is given in ESI Section 2. To demonstrate the applicability of this model to 
a real elastomer, we compare this model with experimental results. Again, due to the lack of exact information about the cross-linked networks, the parameters $G_{c}, G_{e}$ and $\lambda_{\max }$ are calibrated through experimental results. While the $N_{v}, n_{v}, b, a_{\mathrm{pp}}$ and $\tau_{d}$ for the free chains are informed and determined by our large scale coarse-grained molecular simulations (cf. Tab. 3) (Li et al., 2012b). Thus, the viscous energy dissipation during the loading and unloading process is predicted by our proposed constitutive model.

Amnuaypornsri et al. (2012) have studied the stress-strain behaviors of un-vulcanized and vulcanized NRs. The un-vulcanized NRs have been uniaxially stretched at different speeds, from 5 to $40 \mathrm{~mm} / \mathrm{min}$ (corresponding to strain rates 0.006 to $0.05 \mathrm{~s}^{-1}$ ), during extension and retraction. The corresponding stressstrain curves are reproduced by dashed lines in Fig. 10. At different strain rates, the un-vulcanized NR shows elastic deformation at the beginning, followed by strain-softening and strain-hardening (upturn) at large strain. The modulus and stress upturns at large strains increase as the strain rate increases, suggesting viscous behavior of un-vulcanized NRs, which is attributed to the entanglements between different rubber chains. As the un-vulcanized NR does not have any chemical cross-linkings, it behaves like an elastic fluid (Amnuaypornsri et al., 2008, 2012). According to experimental observations of Amnuaypornsri et al. (2012), polyisoprene in un-vulcanized NR have different functional chemical groups at both ends, for example, the polymerization initiating end ( $\omega$-terminal) and the polymerization terminating end ( $\alpha$-terminal). The structure of the $\omega$-terminal has not yet well-understood, and the structure of the $\alpha$-terminal has been determined as the mono or diphosphate with acyl-glycerol or phospholipid (Toki et al., 2013). Therefore, the phospholipids, proteins, and metal ions in un-vulcanized NR can react with the $\omega$-terminal, forming hydrogen bonds, and link with the $\alpha$-terminal through fatty acids. Under this situation, the functional groups create a pseudo end-linked network within the un-vulcanized NR, although the molecular weight between cross-linkers is identical to the molecular weight of the whole chain.

As demonstrated in Fig. 10, the experimental results on the stress-strain curves of un-vulcanized NR are compared with our model. We find that all the experimental results can be well reproduced through our constitutive model, except for at high strain rates $\left(0.05 \mathrm{~s}^{-1}\right)$; there are several reasons for this issue. First, all the material parameters for the viscous part are predicted by our coarse-grained molecular simulations (Li et al., $2012 \mathrm{~b}$ ). Due to the lack of information about the cross-linked network, such as cross-linking density and molecular weight between cross-linkages, the related parameters $G_{c}, G_{e}$ and $\lambda_{\max }$ are calibrated through these experimental results. They may not accurately reflect the microstructural information of un-vulcanized NR. Second, according to experimental studies on un-vulcanized NR, it contains $6 \%$ of nonrubber components, e.g. proteins, metal ions, phospholipids and carbohydrates. These impurities serve as the cross-linkers with functionality varying from two to five (Tangpakdee and Tanaka, 1998). They also have the tendency to form micelles together. Therefore, the un-vulcanized NR is an in-homogeneous material according to these experimental observations (Karino et al., 2007; Toki et al., 2008). However, our model assumes that the NR behaves isotropically and homogeneously. According to the above discussion, our constitutive model may not be able to fully capture the stress-strain curves of un-vulcanized NR under different strain rates. Note that if the hyperelastic stress is given by the Arruda-Boyce model, the strain softening behavior cannot be captured, as shown in Fig. S2b of ESI.

The stress-strain behaviors of peroxide vulcanized ${ }^{5} \mathrm{NR}$ at different stretch speeds of 5 to $40 \mathrm{~mm} / \mathrm{min}$ are given in Fig. 11a. In contrast to the un-vulcanized NR, the strain rate effect on the peroxide vulcanized NR is negligible. The hysteresis between the loading and unloading stage is rather small, compared with the unvulcanized NR, indicating the viscous effect has been greatly reduced after vulcanization. Again, the stress-

\footnotetext{
${ }^{5}$ The peroxide vulcanization forms cross-linking (carbon-carbon bond) between adjacent polyisoprene chains through radicals without any additional ingredients. However, the classical sulfur vulcanization requires zinc oxide, stearic acid and an accelerator during cross-linking.
} 

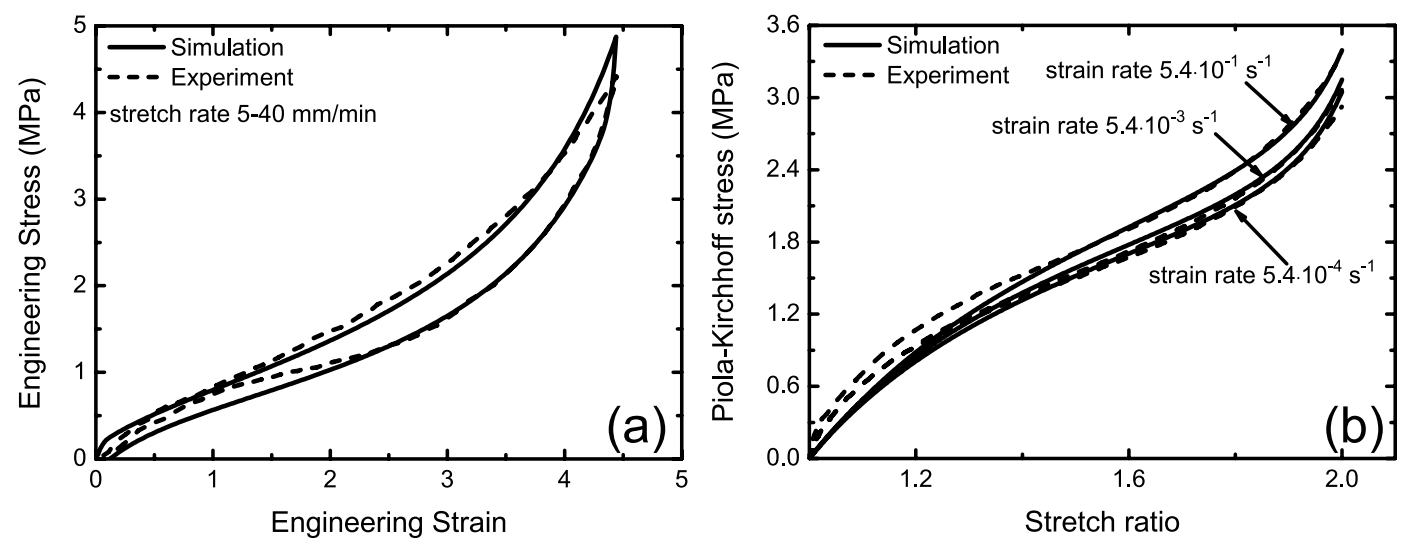

Figure 11: Effect of stain rate on uniaxial tension behaviors of elastomers at room temperature: (a) peroxide vulcanized NR and (b) polymer B186. In (a), the stretch speeds 5 to $40 \mathrm{~mm} / \mathrm{min}$ correspond to strain rates 0.006 to $0.05 \mathrm{~s}^{-1}$, and these curves collapse onto a single curve. In (b), only the loading parts of stress-strain curves have been reported. Thus, the hysteresis between loading and unloading stages are unknown. The experimental results (dashed lines) in (a) and (b) are taken from Amnuaypornsri et al. (2012) and Miehe and Göktepe (2005), respectively. The material parameters entering our constitutive model (solid lines) are collected in Tab. 3.

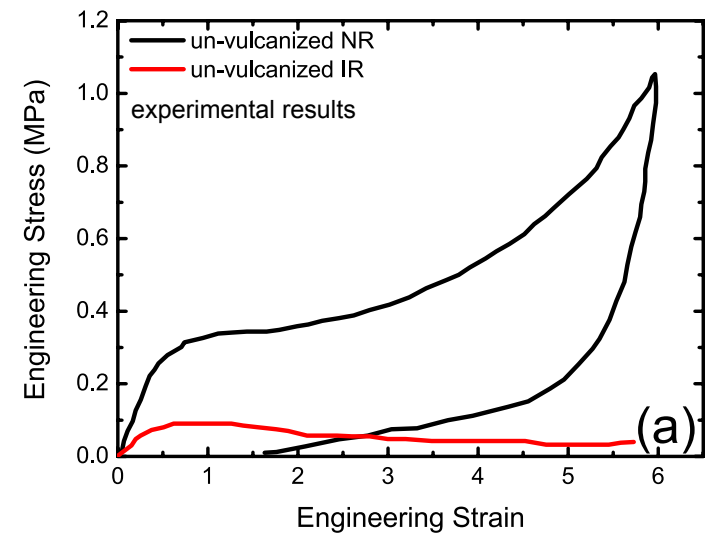

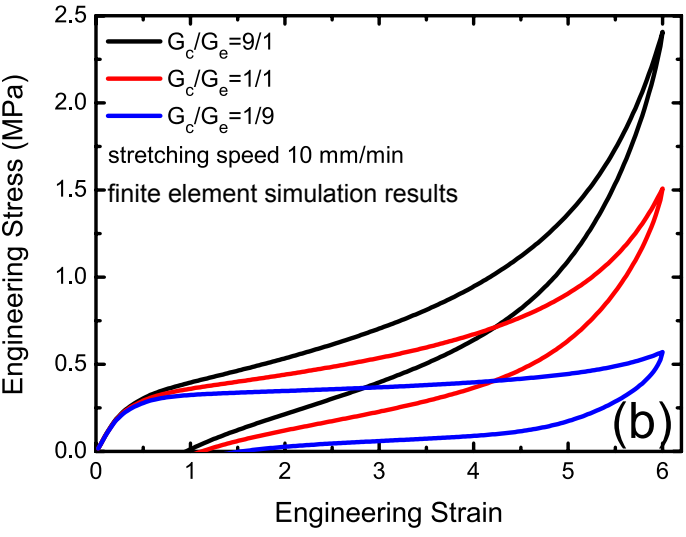

Figure 12: (a) Experimental results on stress-strain curves of un-vulcanized NR and IR under uniaxial tension (strain rate $0.007 \mathrm{~s}^{-1}$ ) at room temperature. (b) Simulation results for effect of ratio $G_{c} / G_{e}$ on stress-strain curves of elastomers. The experimental results in (a) are taken from Toki et al. (2013). In (b), the material parameters in the constitutive model are given in Tab. 3 for un-vulcanized NR. The sum $G_{c}+G_{e}$ is fixed while the ratio $G_{c} / G_{e}$ is changed during the simulation.

The proposed constitutive model can not only be applied to study the viscoelastic properties of unvulcanized and vulcanized rubbers, it can also be used to explain some of the unusual experimental observa- 
tions. For example, the mechanical properties of un-vulcanized NR and synthetic cis-1,4 polyisoprene (IR) have been studied by Toki et al. (2013), as given in Fig. 12a. Although the un-vulcanized NR and IR have the same component, cis-1,4 polyisoprene, they display very different mechanical behaviors. Un-vulcanized NR demonstrates a hysteresis and an elastic response like rubber, as discussed previously. On the other hand, the un-vulcanized IR shows yield stress and flow behavior, since it is in the melt state (typical glass transition temperature $T_{g}=-67^{\circ} \mathrm{C}$ ). As aforementioned, the un-vulcanized NR have functional groups at the ends of the chain, which can interact with the phospholipids, proteins, and metal ions, forming a pseudo end-linked network. Thus, the behavior of un- vulcanized NR is very close to that of rubber due to the pseudo crosslinking. However, the un-vulcanized IR only has entanglements without additional constraints, indicating the contribution of $G_{c}$ is negligible. Therefore, the polymer chains may slide at entanglements during deformation at the melt temperature, demonstrating flow behavior. These behaviors can be easily duplicated by our constitutive model as the effects of cross-linking and entanglement are controlled by $G_{c}$ and $G_{e}$ in the hyperelastic part, respectively. As described in Fig. 12b, when the ratio $G_{c} / G_{e}$ is large, the polymer exhibits large hysteresis with strain-hardening, reminiscent of the behavior of un-vulcanized NRs. On the contrary, the polymer can easily yield and behave like a fluid, similar to un-vulcanized IR, when the ratio $G_{c} / G_{e}$ is greatly reduced. In short, the behavior of un-vulcanized NR and IR polymers are reproduced within the domains of large and small ratios $G_{c} / G_{e}$, respectively, in our constitutive model. Note that the ratio $G_{c} / G_{e}$ governs the strain-softening behavior of elastomers. With $G_{c} / G_{e} \ll 1$, the tangent modulus becomes extremely small for a large portion of the stress-strain curve after an initial elastic deformation, without significant strain-hardening behavior. For $G_{c} / G_{e} \gg 1$, the strain-softening is significantly diminished and strain-hardening is more pronounced.

Besides the ratio $G_{c} / G_{e}$, the remaining parameters of our constitutive model also play very important roles in the mechanical behavior of elastomers. Here we perform a parametric study to demonstrate their influences. Fig. 13a shows the effect of the initial modulus, which is the sum of the cross-link $G_{c}$ and entanglement $G_{e}$ moduli, depending on the cross-link and entanglement densities, respectively. The stressstrain curves are vertically shifted up upon increasing the initial modulus $G_{c}+G_{e}$. The onset of strain hardening is determined by $\lambda_{\max }$, which is mainly affected by the polymerization degree of strands between cross-linkages. This is demonstrated in Fig. 13b. The onset and amplitude of stress during strain hardening is greatly influenced by $\lambda_{\max }$ while the rest of the stress-strain curve remains basically unaffected. Since $G_{c}, G_{e}$ and $\lambda_{\max }$ are parameters from the hyperelastic part, they do not affect the viscous energy loss of elastomers. To further explore how the parameters in our constitutive model can change the viscous response of elastomers, we consider another two parameters in the viscous part: the number of free chains per unit volume $n_{v}$ and the disentanglement time $\tau_{d 0}$ in the absence of deformation. As we can see from Fig. 13c-d, increasing $n_{v}$ or $\tau_{d 0}$ results in gradual enlargement of the hysteresis between the loading and unloading curves, indicating an enhanced amount of energy dissipation.

\section{Concluding Remarks}

To better understand the viscoelastic behaviors of elastomers, they have been decomposed into a crosslinked network with superimposed free chains. The hyperelastic and viscous behaviors of elastomers are attributed to the nonlinear deformation of a cross-linked network and diffusion of free chains, respectively. Large scale molecular simulations were performed to reveal the detailed physical mechanisms of the crosslinked network and free chains under uniaxial tension, simple shear and equal-biaxial tension. For the cross-linked network, its stress response is attributed to the cross-linking and entanglement. Therefore, the classical Arruda-Boyce model (Arruda and Boyce, 1993) is not applicable as it does not include the effect of entanglements. The recently developed non-affine network model (Davidson and Goulbourne, 2013), 

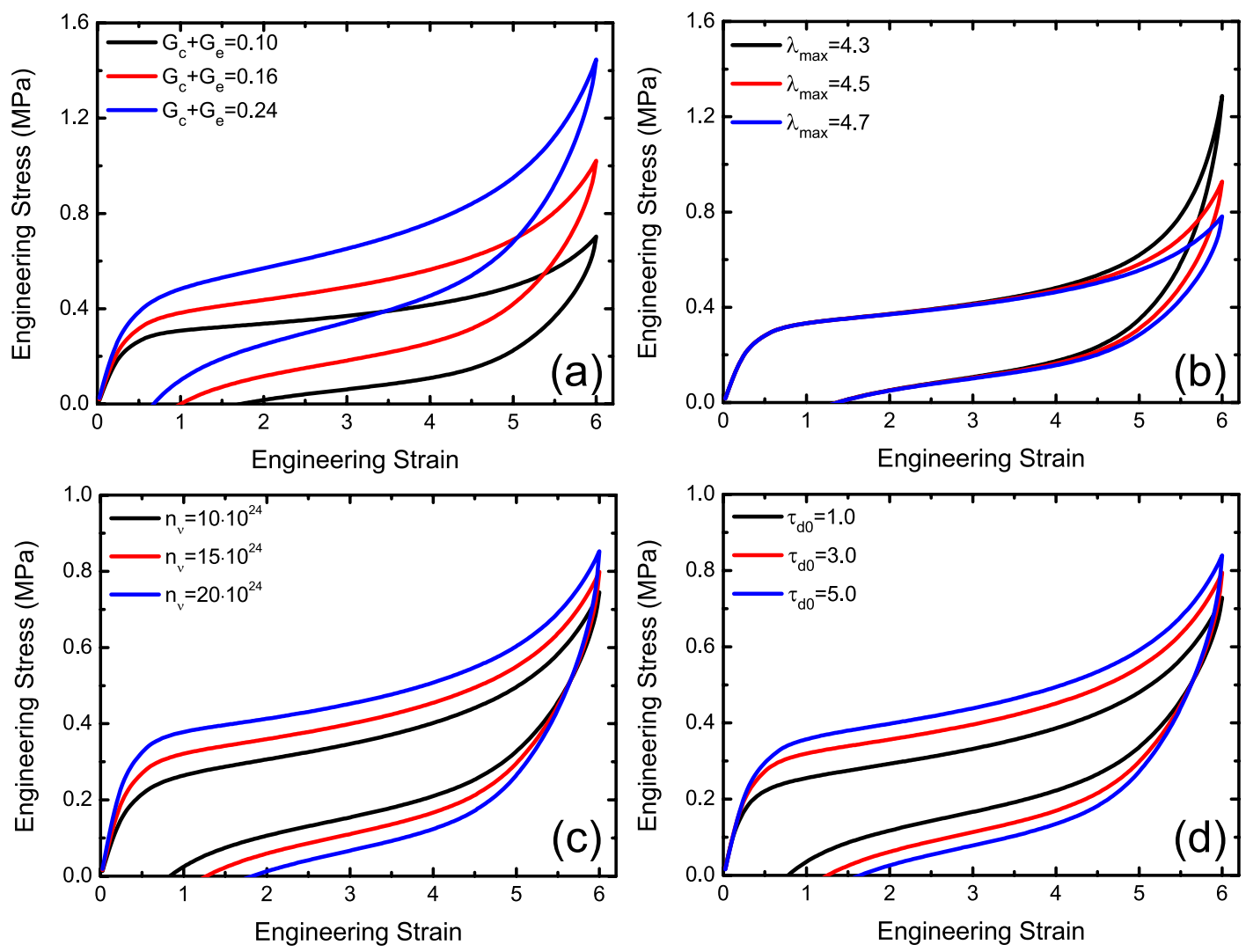

Figure 13: Effect of varying model parameters on the predicted uniaxial stress-strain curves of elastomers: (a) initial modulus $G_{c}+G_{e}$, (b) chain extensibility $\lambda_{\max }$, (c) number of free chains per unit volume $n_{v}$ and (d) equilibrium disentanglement time $\tau_{d 0}$. Unless stated, the material parameters in the constitutive model are given in Tab. 3 for un-vulcanized NR.

simultaneously considering the contributions of cross-linking and entanglement, is identified as the most proper continuum model to describe the hyperelastic behaviors of elastomers. For the free chains, the primitive chain length $L_{\mathrm{pp}}$ and tube diameter $a_{\mathrm{pp}}$ are found to be dependent on the applied deformations in the molecular simulations, which can be captured by our theoretical formulations based on an affine deformation assumption. Based on these observations, the viscous contribution of free chains can be reformulated according to the classical tube model proposed by Doi and Edwards (1986), which is named as the updated tube model. Combining the non-affine network model with the updated tube model, a new constitutive model has been proposed for studying the finite strain viscoelastic behavior of elastomers. This new model is found to be able to capture the mechanical responses of un-vulcanized and vulcanized rubber under uniaxial loading and unloading with different strain rates. Moreover, parametric studies have been performed based on the proposed constitutive model, which demonstrate that different viscoelastic behaviors can be achieved through tuning these physical parameters.

Finally, we would like to emphasize the important role played by the molecular simulations during the development of the proposed constitutive model. These molecular simulations are very useful for determining the material law parameters, such as Kuhn length $b$, tube diameter $a_{\mathrm{pp} 0}$, disentanglement time $\tau_{d}$, for 
Table 3: Material parameters used in our constitutive model for finite strain viscoelasticity of elastomers: cross-linking modulus $G_{c}$, entanglement modulus $G_{e}$, maximum chain extensibility $\lambda_{\max }$, number of free chains per unit volume $n_{v}$, polymerization degree of free chains $N_{v}$, Kuhn length $b$, tube diameter $a_{\mathrm{pp} 0}$ and disentanglement time $\tau_{d 0}$ of the reference (equilibrium) state. The fractional order $\alpha$ is taken to be 0.5 in our numerical simulations. The values of $n_{v}, N_{v}, b, a_{\mathrm{pp} 0}$ and $\tau_{d 0}$ are taken from our coarse-grained molecular simulations (Li et al., 2012b). $G_{c}, G_{e}$ and $\lambda_{\max }$ are calibrated through experimental stress-strain curves, duo to the lack of exact information about cross-linked networks.

\begin{tabular}{ccccccccc}
\hline \hline Polymer & $\begin{array}{c}G_{c} \\
\mathrm{MPa}\end{array}$ & $\begin{array}{c}G_{e} \\
\mathrm{MPa}\end{array}$ & $\lambda_{\max }$ & $\begin{array}{c}n_{v} \\
\mathrm{~cm}^{-3}\end{array}$ & $N_{v}$ & $b$ & $a_{\mathrm{pp} 0}$ & $\tau_{d 0}$ \\
& 0.024 & 0.096 & 4.65 & $16.0 \times 10^{24}$ & 5000 & 0.67 & 6.5 & 3.6 \\
un-vulcanized NR & 0.05 & $\mathrm{~nm}$ & $\mathrm{~s}$ \\
vulcanized NR & 0.34 & 0.02 & 4.0 & $9.0 \times 10^{24}$ & 5000 & 0.67 & 6.5 & 4.378 \\
polymer B186 & 0.1 & 1.5 & 1.355 & $16.0 \times 10^{24}$ & 5000 & 0.67 & 6.5 & 1.5 \\
\hline \hline
\end{tabular}

the viscous part of free chains. Besides, the molecular simulations have been used to verify the underlying assumptions and reveal new physical mechanisms of the continuum model, which are the most important elements during the development of the constitutive model. For example, the capability of the non-affine network model (Davidson and Goulbourne, 2013) has been tested by our large scale molecular simulations. Through these simulations, the contribution of polymer chain entanglements in the non-affine network model has been confirmed, which has not been appreciated before. Note that it is difficult to confirm the entanglement contribution through experiments, as it requires precisely controlled microstructures of elastomers, such as cross-link density, polymerization degree between cross-linkages and entanglements per chain. The molecular simulations are also used to extend the tube theory to the finite deformation regime. Although we have theoretically developed the deformation-dependent expressions for the tube diameter and primitive chain length, it is important to test these assumptions, as they are the key to understand the viscous energy dissipation during the finite deformation stage. In short, we believe that the current molecular simulations play very important roles in understanding and developing the state-of-art continuum theories for hyperelasticity and viscosity.

Acknowledgment We are grateful to Jacob Smith for critical reading of the manuscript and helpful discussions. Y.L. and W.K.L. warmly thank the support from AFOSR grant No. FA9550-14-1-0032. S.T. thanks NSF of Chongqing (Project No. 0211002431039) and NSF of China (Project No. 11472065). M.K. acknowledges support by the Swiss National Science Foundation through grant 200021_156106. This research was supported in part through the computational resources and staff contributions provided for the Quest high performance computing facility at Northwestern University which is jointly supported by the Office of the Provost, the Office for Research, and Northwestern University Information Technology.

\section{References}

Amnuaypornsri, S., Sakdapipanich, J., Toki, S., Hsiao, B.S., Ichikawa, N., Tanaka, Y., 2008. Strain-induced crystallization of natural rubber: effect of proteins and phospholipids. Rubber Chem. Techn. 81, 753-766.

Amnuaypornsri, S., Toki, S., Hsiao, B.S., Sakdapipanich, J., 2012. The effects of endlinking network and entanglement to stress-strain relation and strain-induced crystallization of un-vulcanized and vulcanized natural rubber. Polymer 53, 3325-3330.

Arruda, E.M., Boyce, M.C., 1993. A three-dimensional constitutive model for the large stretch behavior of rubber elastic materials. J. Mech. Phys. Solids 41, 389-412. 
Bergström, J., Boyce, M., 1998. Constitutive modeling of the large strain time-dependent behavior of elastomers. J. Mech. Phys. Solids 46, 931-954.

Craiem, D., Rojo, F., Atienza, J., Guinea, G., Armentano, R.L., 2008. Fractional calculus applied to model arterial viscoelasticity. Latin Amer. Appl. Res. 38, 141-145.

Davidson, J.D., Goulbourne, N., 2013. A nonaffine network model for elastomers undergoing finite deformations. J. Mech. Phys. Solids 61, 1784-1797.

De Gennes, P.G., 1979. Scaling concepts in polymer physics. Cornell University Press, New York.

Doi, M., Edwards, S.F., 1986. The theory of polymer dynamics. Clarendon Press, Oxford.

Edwards, S., 1967. The statistical mechanics of polymerized material. Proc. Phys. Soc. 92, 9-16.

Edwards, S., Vilgis, T., 1986. The effect of entanglements in rubber elasticity. Polymer 27, 483-492.

Edwards, S., Vilgis, T.A., 1988. The tube model theory of rubber elasticity. Rep. Progr. Phys. 51, 243.

Fetters, L., Lohse, D., Richter, D., Witten, T., Zirkel, A., 1994. Connection between polymer molecular weight, density, chain dimensions, and melt viscoelastic properties. Macromolecules 27, 4639-4647.

Govindjee, S., Reese, S., 1997. A presentation and comparison of two large deformation viscoelasticity models. J. Eng. Mater. Technol. 119, 251-255.

Govindjee, S., Simo, J.C., 1992. Mullins effect and the strain amplitude dependence of the storage modulus. Int. J. Solids Struct. 29, 1737-1751.

Heinrich, M., Pyckhout-Hintzen, W., Allgaieer, J., Richter, D., Straube, E., McLeish, T.C.B., Wiedenmann, A., Blackwell, R.J., Read, D.J., 2004. Small-angle neutron scattering study of the relaxation of a melt of polybutadiene H-polymers following a large step strain. Macromolecules 37, 5054-5064.

Hoy, R.S., Foteinopoulou, K., Kröger, M., 2009. Topological analysis of polymeric melts: Chain-length effects and fast-converging estimators for entanglement length. Phys. Rev. E 80, 031803.

Kaliske, M., Heinrich, G., 1999. An extended tube-model for rubber elasticity: statistical-mechanical theory and finite element implementation. Rubber Chem. Techn. 72, 602-632.

Karino, T., Ikeda, Y., Yasuda, Y., Kohjiya, S., Shibayama, M., 2007. Nonuniformity in natural rubber as revealed by small-angle neutron scattering, small-angle $\mathrm{x}$-ray scattering, and atomic force microscopy. Biomacromolecules 8, 693-699.

Keck, J., Miehe, C., 1997. An Eulerian overstress-type viscoplastic constitutive model in spectral form. formulation and numerical implementation. Comput. Plast. Fund. Appl. 1, 997-1003.

Keshavarz, M., Engelkamp, H., Xu, J., Braeken, E., Otten, M.B.J., Uji, H., Schwartz, E., Koepf, M., Vananroye, A., Vermant, J., Nolte, R.J.M., De Schryver, F.C., Maan, J.K., Hofkens, J., Christianen, P.C.M., Rowan, A.E., 2015. Nanoscale study of polymer dynamics. Preprint .

Kröger, M., 2005. Shortest multiple disconnected path for the analysis of entanglements in two-and threedimensional polymeric systems. Comput. Phys. Commun. 168, 209-232. 
Kröger, M., Loose, W., Hess, S., 1993. Rheology and structural changes of polymer melts via nonequilibrium molecular dynamics. J. Rheol. 37, 1057-1079.

Kröger, M., 2015. Simple, admissible, and accurate approximants of the inverse Langevin and Brillouin functions, relevant for strong polymer deformations and flows. J. Non-Newtonian Fluid Mech. 223, 77 87.

Le Tallec, P., Rahier, C., Kaiss, A., 1993. Three-dimensional incompressible viscoelasticity in large strains: formulation and numerical approximation. Comput. Meth. Appl. Mech. Eng. 109, 233-258.

Li, Y., Abberton, B.C., Kröger, M., Liu, W.K., 2013. Challenges in multiscale modeling of polymer dynamics. Polymers 5, 751-832.

Li, Y., Kröger, M., Liu, W.K., 2011. Primitive chain network study on uncrosslinked and crosslinked cispolyisoprene polymers. Polymer 52, 5867-5878.

Li, Y., Kröger, M., Liu, W.K., 2012a. Nanoparticle effect on the dynamics of polymer chains and their entanglement network. Phys. Rev. Lett. 109, 118001.

Li, Y., Tang, S., Abberton, B.C., Kröger, M., Burkhart, C., Jiang, B., Papakonstantopoulos, G.J., Poldneff, M., Liu, W.K., 2012b. A predictive multiscale computational framework for viscoelastic properties of linear polymers. Polymer 53, 5935-5952.

Likhtman, A.E., McLeish, T.C., 2002. Quantitative theory for linear dynamics of linear entangled polymers. Macromolecules 35, 6332-6343.

Lion, A., 1996. A constitutive model for carbon black filled rubber: experimental investigations and mathematical representation. Contin. Mech. Thermodyn. 8, 153-169.

Lion, A., 1997. On the large deformation behaviour of reinforced rubber at different temperatures. J. Mech. Phys. Solids 45, 1805-1834.

Lubliner, J., 1985. A model of rubber viscoelasticity. Mech. Res. Commun. 12, 93-99.

McLeish, T., 2002. Tube theory of entangled polymer dynamics. Adv. Phys. 51, 1379-1527.

Meissner, B., Matějka, L., 2003. A Langevin-elasticity-theory-based constitutive equation for rubberlike networks and its comparison with biaxial stress-strain data. part I. Polymer 44, 4599-4610.

Meral, F., Royston, T., Magin, R., 2010. Fractional calculus in viscoelasticity: an experimental study. Commun. Nonlinear Sci. Numer. Simul. 15, 939-945.

Miehe, C., Göktepe, S., 2005. A micro-macro approach to rubber-like materials. part ii: the micro-sphere model of finite rubber viscoelasticity. J. Mech. Phys. Solids 53, 2231-2258.

Miehe, C., Göktepe, S., Lulei, F., 2004. A micro-macro approach to rubber-like materialsart I: the non-affine micro-sphere model of rubber elasticity. J. Mech. Phys. Solids 52, 2617-2660.

Mooney, M., 1940. A theory of large elastic deformation. J. Appl. Phys. 11, 582-592.

Müller, S., Kästner, M., Brummund, J., Ulbricht, V., 2011. A nonlinear fractional viscoelastic material model for polymers. Comput. Mater. Sci. 50, 2938-2949. 
Ogden, R., 1972. Large deformation isotropic elasticity-on the correlation of theory and experiment for incompressible rubberlike solids. Proc. Royal Soc. London A 326, 565-584.

Ogden, R.W., 1997. Non-linear elastic deformations. Courier Corporation.

Ott, M., Pérez-Aparicio, R., Schneider, H., Sotta, P., Saalwächter, K., 2014. Microscopic study of chain deformation and orientation in uniaxially strained polymer networks: NMR results versus different network models. Macromolecules 47, 7597-7611.

Pyckhout-Hintzen, W., Westermann, S., Wischnewski, A., Monkenbusch, M., Richter, D., Straube, E., Farago, B., Lindner, P., 2013. Direct observation of nonaffine tube deformation in strained polymer networks. Phys. Rev. Lett. 110, 196002.

Reese, S., Govindjee, S., 1998. A theory of finite viscoelasticity and numerical aspects. Int. J. Solids Struct. $35,3455-3482$.

Rivlin, R., 1948. Large elastic deformations of isotropic materials. iv. further developments of the general theory. Phil. Trans. Royal Soc. LondonA 241, 379-397.

Rouse Jr, P.E., 1953. A theory of the linear viscoelastic properties of dilute solutions of coiling polymers. J. Chem. Phys. 21, 1272-1280.

Rubinstein, M., Panyukov, S., 1997. Nonaffine deformation and elasticity of polymer networks. Macromolecules 30, 8036-8044.

Rubinstein, M., Panyukov, S., 2002. Elasticity of polymer networks. Macromolecules 35, 6670-6686.

Schiessel, H., Metzler, R., Blumen, A., Nonnenmacher, T., 1995. Generalized viscoelastic models: their fractional equations with solutions. J. Phys. A 28, 6567-6584.

Schweizer, K.S., Curro, J.G., 1997. Integral equation theories of the structure, thermodynamics, and phase transitions of polymer fluids. Adv. Chem. Phys. 98, 1-142.

Sidoroff, F., 1974. Un modèle viscoélastique non linéaire avec configuration intermédiaire. Journal de Mécanique 13, 679-713.

Simo, J., 1987. On a fully three-dimensional finite-strain viscoelastic damage model: formulation and computational aspects. Comp. Meth. Appl. Mech. Eng. 60, 153-173.

Stephanou, P.S., Baig, C., Tsolou, G., Mavrantzas, V.G., Kröger, M., 2010. Quantifying chain reptation in entangled polymer melts: Topological and dynamical mapping of atomistic simulation results onto the tube model. J. Chem. Phys. 132, 124904.

Straube, E., Urban, V., Pyckhout-Hintzen, W., Richter, D., Glinka, C., 1995. Small-angle neutron scattering investigation of topological constraints and tube deformation in networks. Phys. Rev. Lett. 74, 4464.

Tang, S., Greene, M.S., Liu, W.K., 2012. Two-scale mechanism-based theory of nonlinear viscoelasticity. J. Mech. Phys. Solids 60, 199-226.

Tangpakdee, J., Tanaka, Y., 1998. Branching in natural rubber. Journal of Rubber Research 1, 14-21. 
824 Toki, S., Burger, C., Hsiao, B.S., Amnuaypornsri, S., Sakdapipanich, J., Tanaka, Y., 2008. Multi-scaled 825 microstructures in natural rubber characterized by synchrotron $\mathrm{X}$-ray scattering and optical microscopy. J. 826 Polym. Sci., Part B: Polym. Phys. 46, 2456-2464.

${ }_{827}$ Toki, S., Che, J., Rong, L., Hsiao, B.S., Amnuaypornsri, S., Nimpaiboon, A., Sakdapipanich, J., 2013. ${ }_{828}$ Entanglements and networks to strain-induced crystallization and stress-strain relations in natural rubber 829 and synthetic polyisoprene at various temperatures. Macromolecules 46, 5238-5248.

830 Watanabe, H., 1999. Viscoelasticity and dynamics of entangled polymers. Progr. Polym. Sci. 24, 1253-1403.

${ }_{831}$ Yeoh, O., 1993. Some forms of the strain energy function for rubber. Rubber Chem. Techn. 66, 754-771. 